Егорова О.В., Жаворонок С.И., Курбатов А.С. О вариационных уравнениях расширенной теории N-го порядка упругих оболочек и их приложении к некоторым задачам динамики // Вестник ПНИПУ. Механика. - 2015. - № 2. C. 36-59. DOI: 10.15593/perm.mech/2015.2.03

Egorova O.V., Zhavoronok S.I., Kurbatov A.S. An application of various n-th order shell theories to normal waves propagation problems. PNRPU Mechanics Bulletin. 2015. No. 2. Pp. 36-59. DOI: 10.15593/perm.mech/2015.2.03

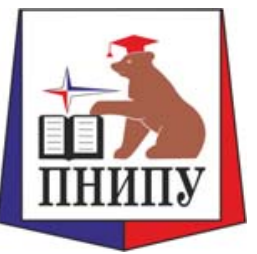

ВЕСТНИК ПНИПУ. МЕХАНИКА

№ 2, 2015

PNRPU MECHANICS BULLETIN

http://vestnik.pstu.ru/mechanics/about/inf/

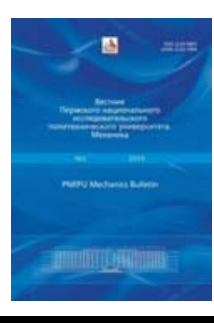

DOI: $10.15593 /$ perm.mech/2015.2.03

УДК 539.3

О ВАРИАЦИОННЫХ УРАВНЕНИЯХ РАСШИРЕННОЙ ТЕОРИИ N-ГО ПОРЯДКА УПРУГИХ ОБОЛОЧЕК И ИХ ПРИЛОЖЕНИИ К НЕКОТОРЫМ ЗАДАЧАМ ДИНАМИКИ

\author{
О.В. Егорова ${ }^{1}$, С.И. Жаворонок ${ }^{2}$, А.С. Курбатов ${ }^{3}$ \\ ${ }^{1}$ Московский авиационный институт (Национальный исследовательский университет) \\ ${ }^{2}$ Институт прикладной механики Российской академии наук (ИПРИМ РАН) \\ ${ }^{3}$ Исследовательский центр им. М.В. Келдыша
}

\section{O CTATbE}

Получена: 27 марта 2015 г.

Принята: 6 мая 2015 г.

Опубликована: 30 июня 2015 г.

Ключевые слова:

оболочки толстостенные, аналитическая механика континуальных систем, формализм Лагранжа, слои упругие,

волны нормальные, скорости групповые, явление «обратной волны», теория пластин $\mathrm{N}$-го порядка

\begin{abstract}
АННОТАЦИЯ
Одной из основных задач теории оболочек средней и большей толщины является моделирование высокочастотных колебаний и распространения волн. Во многих практически важных случаях, возникающих в инженерной практике аэрокосмической отрасли, такие процессы не могут быть адекватно описаны традиционными теориями, основанными на методе гипотез. Требуется разработка моделей, учитывающих, помимо векторов перемещения и поворота, высшие степени свободы и, следовательно, взаимодействие движений в тангенциальном и трансверсальном направлениях.

В данной статье рассматриваются некоторые варианты теории оболочек $\mathrm{N}$-го порядка, принадлежащей к классу теорий И.Н. Векуа и предложенной А.А. Амосовым. Применяемые варианты теории основаны на вариационном формализме аналитической механики континуальных систем со связями. Модель оболочки представляет собой поверхность с заданными на ней множеством обобщенных координат (переменных поля) и поверхностной плотностью лагранжиана. Уравнения движения оболочки имеют вид обобщенных уравнений Лагранжа второго рода. Удовлетворение краевых условий на лицевых поверхностях обеспечивается в расширенном варианте теории. Краевые условия переносятся на базисную поверхность и записываются относительно переменных поля, образуя уравнения связей в континуальной механической системе.

Поставлена однородная задача о распространении нормальных волн в плоском упругом слое и рассмотрено ее решение на базе теории пластин N-го порядка. Продолжено исследование описания теориями низших порядков второй распространяющейся продольной моды, отличающейся эффектом «обратной волны» - различными знаками фазовой и групповой скоростей в диапазоне малых волновых чисел. На базе решения спектральной задачи получены формы второй распространяющейся продольной моды при различных значениях волнового числа, изучена эволюция формы, приводятся оценки аппроксимации формы волны на базе теорий пластин различного порядка. Сделаны выводы о точности описания «обратной волны» расширенными и элементарными теориями пластин, основанными на ортогональных разложениях вектора перемещения.
\end{abstract}

(c) Егорова Ольга Владимировна - кандидат фризико-математических наук, доцент, e-mail: janus_olga@mail.ru Жаворонок Сергей Игоревич - кандидат физико-математических наук, доцент, e-mail: zhavor71@mail.ru Курбатов Алексей Сергеевич - кандидат технических наук, старший научный сотрудник, e-mail: defunt@inbox.ru

Olga V. Egorova - PhD in Physical and Mathematical Sciences, Associate Professor, e-mail: janus_olga@mail.ru Sergey I. Zhavoronok - PhD in Physical and Mathematical Sciences, Associate Professor, e-mail: zhavor71@mail.ru Alexey S. Kurbatov - PhD in Technical Sciences, Senior Researcher, e-mail: defunt@inbox.ru 


\title{
THE VARIATIONAL EQUATIONS OF THE EXTENDED N'TH ORDER SHELL THEORY AND ITS APPLICATION TO SOME PROBLEMS OF DYNAMICS
}

\author{
O.V. Egorova', S.I. Zhavoronok ${ }^{2}$, A.S. Kurbatov ${ }^{3}$ \\ ${ }^{1}$ Moscow Aviation Institute (National Research University) \\ ${ }^{2}$ Institute of Applied Mechanics of Russian Academy of Sciences \\ ${ }^{3}$ Federal State Unitary Enterprise Research Centre named after M.V. Keldysh
}

\begin{tabular}{l} 
ARTICLE INFO \\
\hline Received: 27 March 2015 \\
Accepted: 6 May 2015 \\
Published: 30 June 2015 \\
\hline Keywords: \\
thick shells, shell theories \\
of Vekua type, analytical \\
mechanics of continua, \\
Lagrange formalism, elastic layer, \\
normal waves, group velocities, \\
inverse wave effect, \\
plate theory of N'th order
\end{tabular}

\begin{abstract}
One of topical problems of thick shells theory consists in an accurate modelling of high-frequency shell vibrations and wave propagation, as well as in the application of developed mathematical models to engineering problems of structural dynamics. Many observed processes of both periodic and non-steady shell dynamics cannot be adequately simulated on the groundwork of most traditional hypothesis-based shell theories. Therefore the engineering practice requires new shell models considering higher degrees of freedom besides the displacement and rotation vectors and taking into account the interference of tangential and transversal motions.

Here a variant of the N'th order shell model of I.N. Vekua type is analyzed. The proposed model improves the A. Amosov's shell theory; it is based on the Lagrange's formalism of analytical mechanics extended to continuum systems, on the dimensional reduction approach, and on the biorthogonal expansion technique. The shell is finally represented by a material surface framed by a set of generalized coordinates (or field variables) and a scalar generator function - the surficial density of the Lagrangian. The dynamic equations for the shell are formulated as generalized Lagrange equations of the second kind.

A propagation of normal waves in a plane elastic layer is considered, the corresponding homogeneous problem of N'th order plate theory is formulated, and its solution analysis is extended. In particular, the investigation of the second longitudinal propagating mode and its modelling by approximated plate theories are expanded. The main feature of the second mode is the "inverse wave" effect, i.e. the opposite signs of the phase and group velocities at small wavenumbers. It was shown earlier that the accurate dispersion curves for both phase frequency and group velocity can be obtained on the basis of the fourth- and fifth-order theories. Using the known solution of the spectral problem of $\mathrm{N}$ 'th order plate theory the eigenfunctions are obtained. The second propagating mode evaluation at the smallest wavenumbers is modelled, the forms of the second propagating wave are investigated, and the approximation of the waveforms at some wavenumbers by plate theories of various orders is estimated. The accuracy of the approximation of the "inverse wave" by some applied theories of various orders based on the orthogonal expansions of displacement vector is discussed.
\end{abstract}

(c) PNRPU

\section{Введение}

Одним из основных элементов конструкций авиационно-космических систем различного назначения является тонкостенная оболочка, подвергающаяся, как правило, интенсивному силовому и тепловому динамическому воздействию со стороны окружающей среды, собственных энергетических установок и оборудования. Разработка перспективной аэрокосмической техники требует качественного анализа особенностей динамических процессов, протекающих в тонкостенных элементах конструкций при высокочастотных вибрациях и распространении волн. В ряде случаев традиционно используемые модели элементов такого типа, адекватно описывающие низкочастотные процессы и притом обладающие компактной формулировкой, становятся непригодными. Такие ситуации возникают, например, при описании высокочастотных вибраций, а также при моделировании распространения и интерференции волн. По мере ускорения процессов и повышения диапазона частот сначала классические теории, основанные на гипотезах КирхгоффаЛява и их уточнения, принимающие во внимание инерцию вращения нормального эле- 
мента, а затем и теории класса Тимошенко-Райсснера-Миндлина, учитывающие сдвиг в трансверсальной плоскости, становятся непригодными. Дальнейшее уточнение модели, как правило, диктует отказ от метода гипотез и перехода к редукции трехмерной задачи механики деформируемого твердого тела.

Исчерпывающее описание как высокочастотных колебаний, так и процессов распространения волн в тонкостенных системах на базе «двумерных» моделей на текущий момент не достигнуто $[1,2]$. Обзоры основных работ, посвященных так называемым «неклассическим» моделям оболочек и их приложению к задачам о колебаниях и опубликованных до 1970-х гг., содержатся в работах [3] и [4]. В течение последних 15-20 лет интерес к подобным теориям оболочек возобновился. В работах $[5,6]$ продемонстрировано существенное влияние трансверсальных нормальных напряжений на динамику слоистых оболочек; статья [7] содержит достаточно подробный аналитический обзор моделей высшего порядка тонкостенных слоистых структур, разработанных до 2002 г, и их практических реализаций на базе метода конечных элементов. Результаты работ [5-7] подтвердили выводы Койтера [8] о необходимости совместного учета трансверсальной сдвиговой и нормальной податливости при уточнении классических моделей оболочек, что представляется возможным в рамках теорий высших порядков. В работе [9] исследовано явление так называемого «толщинного» запирания в теориях оболочек низших порядков, возникающего при предположении об отсутствии деформации изменения толщины оболочки, и показана возможность его исключения при учете высших степеней свободы. В статье [10] обоснована необходимость применения теорий с порядком аппроксимации до четвертого в случае высокочастотных колебаний пластины вне зависимости от толщины. В работе [11], в свою очередь, на основе теорий высших порядков показано, что при высокочастотной вибрации пластины существуют решения типа пограничного слоя, локализующегося вблизи лицевых поверхностей. Эффективность моделей, основанных на разложении по системам специальных функций, продемонстрирована в статье [12] на примере круговой пластины. «Трехмерные» модели на базе степенных разложений были использованы для решения задачи о колебаниях конических оболочек [13]. Другим примером необходимого применения теорий высшего порядка является описание статического деформирования [14], колебаний и потери устойчивости функциональноградиентных пластин $[15,16]$ и оболочек $[17,18]$, применяемых в системах тепловой защиты летательных аппаратов при комбинированном воздействии. В [19-22] теории высших порядков использованы при решении обратных задач нестационарной динамики вязкоупругих композиционных тонкостенных элементов.

Помимо исследования высокочастотных колебаний, теории оболочек, учитывающие высшие степени свободы, требуются при решении таких задач, как изучение краевых и кромочных волн; классическая теория пластин способна описать только первую (фундаментальную) краевую волну, тогда как для учета высших волн необходима трехмерная постановка задачи [23]. Следовательно, усовершенствование моделей деформирования оболочек, учитывающих высшие степени свободы и взаимодействие тангенциальных и трансверсальных движений, является актуальным.

Проблема приведения трехмерной задачи к двумерной оболочки не имеет единственного решения [24] и базируется на различных математических методах [25]. «... Вопросы построения приближенных теорий колебаний стержней, пластин и оболочек... представляют собой часть общей проблемы построения моделей механики сплошных сред, получившей развитие в работах Л.И. Седова» $[4$, с. 6]. Обзору ранних решений проблемы при- 
ведения посвящены работы $[4,24]$. Обычно выделяют методы асимптотического интегрирования уравнений теории упругости [26, 27], прямого построения теории материальных поверхностей $[28,29]$, метод пространственной редукции трехмерной задачи [14-22, $30-43]$ на базе разложений по степенным [14-18, 30] либо специальным функциям [5-7, 9, 10, 19-22, 33-43], непрерывным либо кусочно-гладким (в случае слоистых оболочек) [57], в комбинации с методом Галеркина ([31, 32, 36-38, 42] и др.) или вариационным подходом [например, 24, 33-35, 39-41 и др.].

В данной работе описано совместное приложение метода И.Н. Векуа [31] пространственной редукции трехмерной задачи теории упругости, редукционного подхода А.А. Амосова, использованного ранее в работах [37-43], и формализма аналитической механики континуальных систем [44]. Вариационный формализм построения трехмерной, т.е. способной описывать напряженно-деформированное состояние в областях неприводимости, теории А.А. Амосова оболочек N-го порядка был предложен в работах [45-49]. Модель оболочки формулируется в терминах двумерного многообразия как континуальная система, заданная конфигурационным пространством с $\mathrm{N}$ векторными переменными поля первого рода и определенной на нем поверхностной плотностью функционала Лагранжа. Уравнения движения оболочки строятся на базе формализма [45-48] как обобщенные уравнения Лагранжа второго рода относительно обобщенных усилий [45-48] или переменных поля первого рода [49]. Определяющие уравнения модели N-го порядка анизотропной упругой оболочки приведены в работе [46].

В цитируемых выше статьях [45-49] проблема удовлетворения краевых условий на лицевых поверхностях оболочки рассматривалась на базе «упрощенной», согласно терминологии И.Н. Векуа [31], или «элементарной» теории оболочек N-го порядка [37]. Вариационный метод редукции уравнений трехмерной задачи [44] обеспечивает удовлетворение краевых условий на лицевых поверхностях «в среднем» [37, 45]. В рамках проекционного метода [31] приведения трехмерной задачи теории упругости к двумерной задаче теории оболочек предложены различные приемы, обеспечивающие удовлетворение краевых условий на лицевых поверхностях: метод корректирующего решения [37] или «расширенная» формулировка теории оболочек [37], «метод нормированных моментов» И.Н. Векуа [31], использующего в качестве дополнительных неизвестных остаточные члены рядов компонентов вектора перемещения. Вариационный формализм аналитической механики континуальных систем [44], в свою очередь, позволяет рассматривать краевые условия, перенесенные на базисную поверхность оболочки с ее лицевых поверхностей в процессе редукции модели, в качестве уравнений дополнительных связей, накладываемых на переменные поля первого рода [44], голономных в случае кинематических краевых условий и неголономных в случае силовых краевых условий на лицевых поверхностях. Полученная таким образом вариационная задача с ограничениями в форме равенств решается методом множителей Лагранжа, причем уравнения движения, записанные относительно новых обобщенных сил, включающих слагаемые со множителями, идентичны по форме уравнениям элементарной теории N-го порядка. Разрешение уравнений связей относительно множителей Лагранжа позволяет получить новые определяющие уравнения, учитывающие влияние краевых условий на лицевых поверхностях. Можно показать, что изгибная жесткость пластины в рамках теорий низшего порядка совпадает с цилиндрической жесткостью классической теории без принятия гипотезы о трансверсальной изотропии материала [50, 51] либо применения методов штрафа [9] или преобразования определяющих уравнений [52]. Таким образом, устраняется погреш- 
ность вариационного подхода, согласно [29], где указано, что «...трехмерная энергия учитывает энергию тех движений частиц оболочки, трехмерного тела, которые никак не сказываются на движении собственно двумерной оболочки» $(29$, с. 15$)$, или эффект «толщинного» запирания [9].

Свойства модели, описываемой уравнениями теории $\mathrm{N}$-го порядка $[45,46]$, исследованы в работах [41, 43, 47, 48, 54-57]. В работах [41] и [43] получены оценки сходимости приближенных решений различных задач статики на базе трехмерной модели N-го порядка к известным точным решениям. Работы [47, 48, 54-57], в свою очередь, посвящены решению различных модельных задач динамики. Так как «...наиболее простым методом исследования уравнений динамической теории стержней, пластин и оболочек является метод дисперсионных уравнений» ([4], с. 25), в работах $[47,54,55]$ на базе теории пластин N-го порядка были построены дисперсионные уравнения задачи о распространении нормальных волн в плоском упругом слое. Исследована сходимость спектра при нулевом значении волнового числа (т. е. частот запирания распространяющихся нормальных мод). В статье [56] на основе решения спектральной задачи теории N-го порядка вычислены приближенные формы распространяющихся мод нормальных волн, соответствующие частотам запирания, и их погрешности относительно точных решений [58] по норме евклидова пространства. Кроме того, получены значения фазовых частот в точках равенства соответствующих фазовых скоростей и скорости дилатационной волны $c_{1}$ [56], а также погрешности приближенных форм нормальных мод, соответствующих данным точкам, относительно точного решения (мод Гудьера-Бишопа) в среднем квадратичном. В работе [56] исследованы погрешности мод Ламе.

При исследовании свойств теорий пластин низших порядков особый интерес представляет их способность правильно описывать вторую нормальную продольную моду, распространяющуюся в плоском упругом слое и отличающуюся существованием диапазона волновых чисел $\left[0, \kappa^{*}\right]$ с положительной фазовой и отрицательной групповой скоростью [58]. Физическая возможность возбуждения «обратных» волн, доказанная экспериментально в работе [59], делает данную задачу весьма интересной как с точки зрения практических приложений, в частности, в акустике машин [60], так и в качестве тестовой для анализа различных приближенных моделей. В работах $[47,49]$ на базе элементарных теорий 1-5-го порядков, не учитывающих краевые условия на лицевых поверхностях в форме связей и использующих в качестве базисной системы полиномы Лежандра, построен начальный участок второй дисперсионной ветви, соответствующий «обратной» волне. Показано, что адекватное значение групповой скорости достигается на основе теорий не ниже 4-го порядка. В работе [57] исследовано описание формы второй распространяющейся моды продольных волн в области существования эффекта «обратной волны». Эволюция формы волны описана элементарной теорией пластин 5-го порядка.

Ниже приводится анализ второй распространяющейся моды, построенной на основе решения спектральной задачи в рамках элементарной и расширенной теории 2-5-го порядков. Получены зависимости компонентов вектора перемещения от безразмерной нормальной координаты в диапазоне малых волновых чисел, определяющие эволюцию формы «обратной» нормальной волны. Исследована сходимость решения по мере повышения порядка применяемой теории пластин. Полученные результаты являются основой моделирования распространения волн в тонких телах на базе моделей, являющихся частными случаями теории оболочек $\mathrm{N}$-го порядка. 


\section{1. Формулировка элементарной теории оболочек N-го порядка}

\section{Параметризация геометрии оболочки и ее базисной поверхности}

Пусть оболочка занимает область $V \in R^{3}, \partial V=S_{ \pm} \oplus S_{B} ; S_{ \pm}$- лицевые, $S_{B}$ - боковые поверхности оболочки. Параметризация оболочки в рамках теорий, описываемых в терминах двумерного многообразия, заключается:

1) в параметризации базисной поверхности $S_{0}$, являющейся геометрической моделью оболочки и определяющей формулировку двумерной начально-краевой задачи;

2) в параметризации области $V \in R^{3}$, являющейся ключевым пунктом решения «проблемы приведения» [24] трехмерной задачи к двумерному эквиваленту.

Параметризация $\boldsymbol{S}_{\mathbf{0}}$. Введем криволинейные координаты $\xi^{\beta}, \beta=1,2$ :

$$
\forall M_{0} \in S_{0} \quad \mathbf{r}\left(M_{0}\right)=\mathbf{r}\left(\xi^{\beta}\right)=x^{i}\left(\xi^{\beta}\right) \mathbf{e}_{i},
$$

где $x^{i}$ - декартовы координаты точки $M_{0}$. Здесь и далее по повторяющемуся индексу подразумевается суммирование; латинские индексы пробегают значения 1, 2, 3; греческие-значения 1, 2. Базис $\mathbf{r}_{\alpha}$ и метрика $\mathbf{a}$ касательного расслоения $T_{M} S_{0}$, единичная нормаль $S_{0}$ и тензор кривизны $\mathbf{b}$ определяются обычным образом [31]:

$$
\begin{gathered}
\mathbf{r}_{\alpha}\left(M_{0}\right)=\partial_{\alpha} \mathbf{r}\left(\xi^{\beta}\right)=J_{\alpha}^{i} \mathbf{e}_{i}, \quad J_{\alpha}^{i}=\partial x^{i} / \partial \xi^{\alpha}, \\
a_{\alpha \beta}=\mathbf{r}_{\alpha} \cdot \mathbf{r}_{\beta} ; \quad \mathbf{r}^{\beta}=a^{\alpha \beta} \mathbf{r}_{\alpha}, \quad \mathbf{r}_{\alpha}=a_{\alpha \beta} \mathbf{r}^{\beta}, \quad a^{\alpha \gamma} a_{\gamma \beta}=\delta_{\beta}^{\alpha} ; \quad a=\operatorname{det}\left(a_{\alpha \beta}\right), \\
\mathbf{n}=\left(\mathbf{r}_{1} \times \mathbf{r}_{2}\right) / \sqrt{a} ; \quad b_{\alpha \beta}=-\left[\partial \mathbf{n} / \partial \xi^{\alpha}\right] \cdot \mathbf{r}_{\beta} ; \quad c_{\alpha \beta}=b_{\alpha}^{\gamma} b_{\gamma \beta} .
\end{gathered}
$$

Определим также среднюю $H\left(M_{0}\right)=\frac{1}{2} b_{\alpha}^{\alpha}$ и гауссову $K\left(M_{0}\right)=\operatorname{det}\left(b_{\alpha}^{\beta}\right)$ кривизны $S_{0}$.

Параметризация $\boldsymbol{V}$. Введем нормальную координату $\xi^{3} \in\left[h_{-}, h_{+}\right]$, так, что

$$
\forall M \in V \backslash S_{0} \quad \mathbf{R}(M)=\mathbf{r}\left(M_{0}\right)+\xi^{3} \mathbf{n}\left(M_{0}\right),
$$

где $h=h_{+}-h_{-}-$толщина оболочки. При $\bar{h}=\frac{1}{2}\left(h_{+}-h_{-}\right)=0 S_{0}-$ срединная поверхность. Базис $\mathbf{R}_{\alpha}, \mathbf{n}$ пространственной системы координат $\xi^{1}, \xi^{2}, \xi^{3}$ определен так [31]:

$$
\begin{gathered}
\mathbf{R}_{\alpha}=\partial \mathbf{R}\left(M_{0}, \xi^{3}\right) / \partial \xi^{\alpha}=A_{\alpha \cdot \beta}^{\cdot \beta} \mathbf{r}_{\beta}\left(M_{0}\right) ; \quad A_{\alpha \cdot}^{\cdot \beta}=\delta_{\alpha}^{\beta}-\xi^{3} b_{\alpha}^{\beta} ; \quad \mid A_{\alpha \cdot \bullet}^{\cdot \beta}=1-2 \xi^{3} H+\left(\xi^{3}\right)^{2} K, \\
\mathbf{r}_{\alpha}=A_{\cdot \alpha}^{\beta \cdot} \mathbf{R}_{\beta}, \quad A_{\bullet \alpha}^{\beta \cdot}=\left[1-2 \xi^{3} H+\left(\xi^{3}\right)^{2} K\right]^{-1}\left[\delta_{\alpha}^{\beta}-\xi^{3}\left(2 H \delta_{\alpha}^{\beta}-b_{\beta}^{\alpha}\right)\right] .
\end{gathered}
$$

Условия однозначности параметризации (2) приведены в [47]. Там же введена система координат $\eta^{1}, \eta^{2}, \eta^{3}$, голономным базисом которой являются векторы $\mathbf{r}_{\alpha}, \mathbf{n}$ :

$$
\begin{gathered}
\mathbf{r}_{\alpha}=\frac{\partial \mathbf{R}}{\partial \eta^{\alpha}}=\frac{\partial \xi^{\beta}}{\partial \eta^{\alpha}} \frac{\partial \mathbf{R}}{\partial \xi^{\beta}} \Rightarrow A_{\cdot \alpha}^{\beta \cdot}=\frac{\partial \xi^{\beta}}{\partial \eta^{\alpha}}, \quad A_{\alpha \cdot}^{\cdot \beta}=\frac{\partial \eta^{\beta}}{\partial \xi^{\alpha}} ; \quad \eta^{3}=\xi^{3}, \\
\nabla=\mathbf{R}^{\alpha} \frac{\partial}{\partial \eta^{\alpha}}+\mathbf{n} \frac{\partial}{\partial \eta^{3}}=\mathbf{r}^{\alpha} A_{\cdot \alpha}^{\beta \cdot} \frac{\partial}{\partial \xi^{\beta}}+\mathbf{n} \frac{\partial}{\partial \xi^{3}} .
\end{gathered}
$$


С учетом (3) дисторсия и плотность потенциала записываются в виде [46, 47]

$$
\begin{gathered}
\mathbf{d}=\left(\bar{\nabla}_{\beta} u_{\alpha}-b_{\alpha \beta} u_{3}\right) \mathbf{R}^{\beta} \mathbf{r}^{\alpha}+\left(\bar{\nabla}_{\beta} u_{3}+b_{\beta}^{\alpha} u_{\alpha}\right) \mathbf{R}^{\beta} \mathbf{n}+\partial_{3} u_{\alpha} \mathbf{n} \mathbf{r}^{\alpha}+\partial_{3} u_{3} \mathbf{n n}, \quad \partial_{3} \equiv \partial / \partial \xi^{3}, \\
2 W=\boldsymbol{\sigma}: \mathbf{d}=s^{\alpha \beta}\left(\bar{\nabla}_{\beta} u_{\alpha}-b_{\alpha \beta} u_{3}\right)+s^{3 \beta}\left(\bar{\nabla}_{\beta} u_{3}+b_{\beta}^{\alpha} u_{\alpha}\right)+s^{\alpha 3} \partial_{3} u_{\alpha}+s^{33} \partial_{3} u_{3}, \\
\boldsymbol{\sigma}=s^{\alpha \beta} \mathbf{r}_{\alpha} \mathbf{R}_{\beta}+s^{3 \beta} \mathbf{n} \mathbf{R}_{\beta}+s^{\alpha 3} \mathbf{r}_{\alpha} \mathbf{n}+s^{33} \mathbf{n n},
\end{gathered}
$$

где $\bar{\nabla}_{\alpha}-$ ковариантная производная на двумерном многообразии $S_{0} ; \boldsymbol{\sigma}$ - симметричный тензор напряжения; $u_{i}$ - компоненты вектора перемещения в базисе $\mathbf{r}^{\alpha}, \mathbf{n}$, не зависящем от координаты $\xi^{3}$.

\section{Формулировка модели N-го порядка анизотропной оболочки}

В соответствии с подходом [45-49] модель оболочки N-го порядка - континуальная система на $S_{0} \in R^{3}$, заданная конфигурационным пространством

$$
\Omega=\left\{\mathbf{u}^{(k)}\right\}, \quad i=1 \ldots 3, \quad k=0 \ldots N, \quad \mathbf{u}^{(k)}=\mathbf{u}^{(k)}\left(M_{0}, t\right), \quad M_{0} \in S_{0},
$$

где $t \in\left[t_{0}, t_{1}\right] \subseteq R_{+} \cup\{0\}-$ время, $\mathbf{u}^{(k)}$ - переменные поля I рода [44],

$$
\begin{gathered}
\mathbf{u}(M, t)=\mathbf{u}^{(k)}\left(M_{0}, t\right) \mathrm{p}_{(k)}(\zeta)=\left[u_{\alpha}^{(k)}\left(M_{0}, t\right) \mathbf{r}^{\alpha}\left(M_{0}\right)+u_{3}^{(k)}\left(M_{0}, t\right) \mathbf{n}\left(M_{0}\right)\right] \mathrm{p}_{(k)}(\zeta), \\
u_{i}^{(k)}\left(M_{0}, t\right)=\left(u_{i}\left(M_{0}, \zeta, t\right), \mathrm{p}^{(k)}(\zeta)\right)_{1},
\end{gathered}
$$

где $\zeta=2\left(\xi^{3}-\bar{h}\right) / h \in[-1,1]$ - безразмерная нормальная координата $[45,46] ; \mathrm{p}_{(k)}(\zeta)$, $\mathrm{p}^{(m)}(\zeta)-$ биортогональный базис в гильбертовом пространстве $\aleph[-1,1], k, m \in[0, N] \cap \mathbb{Z}$ :

$$
\left(\mathrm{p}_{(k)}, \mathrm{p}^{(m)}\right)_{1} \equiv \int_{-1}^{1} \mathrm{p}_{(k)}(\zeta) \mathrm{p}^{(m)}(\zeta) d \zeta=0, \quad k \neq m
$$

Здесь и далее подразумевается суммирование по повторяющимся латинским индексам, заключенным в скобки. С учетом (4), (5), (8)-(10) записываются поверхностная и контурная плотности функционала Лагранжа $[45,46]$ :

$$
\begin{gathered}
\Lambda_{S}=\Lambda_{S}\left(u_{\alpha}^{(k)}, u_{3}^{(k)}, \dot{u}_{\alpha}^{(k)}, \dot{u}_{3}^{(k)}, \bar{\nabla}_{\beta} u_{\alpha}^{(k)}, \bar{\nabla}_{\beta} u_{3}^{(k)}\right)=\frac{1}{2} \rho_{(k)}^{(m)}\left(\dot{u}_{(m)}^{\alpha} \dot{u}_{\alpha}^{(k)}+\dot{u}_{(m)}^{3} \dot{u}_{3}^{(k)}\right)+P_{(k)}^{\alpha} u_{\alpha}^{(k)}- \\
-\frac{1}{2}\left[\left[s_{(k)}^{\alpha \beta}\left(\bar{\nabla}_{\beta} u_{\alpha}^{(k)}-b_{\beta \alpha} u_{3}^{(k)}\right)+h^{-1} s_{(k)}^{\alpha 3} D_{(m \cdot)}^{(\cdot k)} u_{\alpha}^{(m)}+s_{(k)}^{3 \beta}\left(\bar{\nabla}_{\beta} u_{3}^{(k)}+b_{\gamma}^{\alpha} u_{\alpha}^{(k)}\right)+h^{-1} s_{(k)}^{33} D_{(m \bullet)}^{(\cdot k)} u_{3}^{(m)}\right],\right. \\
\Lambda_{\Gamma}=q_{B(k)}^{\alpha} u_{\alpha}^{(k)}+q_{B(k)}^{\alpha} u_{\alpha}^{(k)}, \\
\rho_{(k)}^{(m)}\left(M_{0}\right)=\left(\rho h \mathrm{p}_{(k)}, \mathrm{p}^{(m)}\right)_{1}, \quad s_{(k)}^{i j}=\left(h s^{i j}, \mathrm{p}_{(k)}\right)_{1}, \quad P_{(k)}^{i}=F_{(k)}^{i}+\bar{q}_{ \pm}^{i}+(-1)^{k} \bar{q}_{ \pm}^{i} .
\end{gathered}
$$

Обобщенные силы $s_{(k)}^{i j}$ являются коэффициентами биортогонального разложения по системе $\mathrm{p}_{(m)}(\zeta)$ компонентов тензора напряжения, заданного в форме (6). Коэффициенты $q_{B(k)}^{\alpha}$ биортогонального разложения компонентов главного вектора внешних сил на кон- 
туре $\Gamma=\partial S_{0}$, так же как и вектора приведенных внешних сил на поверхности $S_{0}$, введены в работах $[45,46] ; \bar{q}_{ \pm}^{i}$ - компоненты главного вектора сил на лицевых поверхностях $S_{ \pm}$.

Линейные операторы $D_{(m)}^{(\cdot k)}$ определяются следующими соотношениями [45]:

$$
D_{(k \cdot)}^{(\cdot m)}=\left(\frac{d}{d \zeta} \mathrm{p}_{(k)}, \mathrm{p}^{(m)}\right)_{1}
$$

\section{Постановка начально-краевой задачи теории элементарной теории N-го порядка}

В соответствии с вариационным формализмом [45-48] из (11) следуют уравнения движения и естественные краевые условия в следующем виде:

$$
\begin{gathered}
\rho_{(k)}^{(m)} \ddot{u}_{(m)}^{\alpha}=\bar{\nabla}_{\beta} s_{(k)}^{\alpha \beta}-b_{\beta}^{\alpha} s_{(k)}^{3 \beta}-h^{-1} D_{(k \bullet)}^{(\bullet m)} s_{(m)}^{\alpha 3}+P_{(k)}^{\alpha}, \\
\rho_{(k)}^{(m)} \ddot{u}_{(m)}^{3}=\bar{\nabla}_{\beta} s_{(k)}^{3 \beta}+b_{\alpha \beta} s_{(k)}^{\alpha \beta}-h^{-1} D_{(k \bullet)}^{(\bullet m)} s_{(m)}^{33}+P_{(k)}^{3} . \\
\left.\left(s_{(k)}^{i \beta} v_{\beta}-q_{B(k)}^{i}\right) \delta u_{i}^{(k)}\right|_{M_{0} \in \Gamma}=0 ; \quad i=1,2,3 ; \quad \beta=1,2 .
\end{gathered}
$$

Уравнениям движения (14) соответствуют начальные условия

$$
\left.u_{(k)}^{i}\right|_{t=t_{0}}=U_{(k)}^{i} ;\left.\quad \dot{u}_{(k)}^{i}\right|_{t=t_{0}}=V_{(k)}^{i},
$$

где $U_{(k)}^{i}\left(M_{0}\right), V_{(k)}^{i}\left(M_{0}\right)$ - коэффициенты разложения компонентов векторов начального смещения и скорости оболочки как трехмерного тела по системе $\mathrm{p}_{(k)}(\zeta)$ при $t=t_{0}$.

Определяющие уравнения теории $\mathrm{N}$-го порядка $[45,46]$ могут быть записаны относительно переменных поля $u_{\alpha}^{(k)}, u_{3}^{(k)}$ и их ковариантных производных:

$$
s_{(k)}^{i \beta}=\overline{\bar{C}}_{(k m)}^{i \beta j \delta} \bar{\nabla}_{\delta} u_{j}^{(m)}+\bar{C}_{(k m)}^{i \beta j} u_{j}^{(m)} ; \quad s_{(k)}^{i 3}=\bar{C}_{(k m)}^{i 3 j \delta} \bar{\nabla}_{\delta} u_{j}^{(m)}+\bar{C}_{(k m)}^{i 3 j} u_{\gamma}^{(m)} .
$$

Коэффициенты жесткости, входящие в (17), определены в $[45,46]$ так:

$$
\begin{gathered}
\overline{\bar{C}}_{(k m)}^{i \beta \gamma}=b_{\delta}^{\gamma} \overline{\bar{C}}_{(k m)}^{i \beta 3 \delta}+h^{-1} D_{(k \bullet)}^{(\cdot n)} \bar{C}_{(n m)}^{i \beta \gamma 3} ; \quad \overline{\bar{C}}_{(k m)}^{i \beta 3}=-b_{\gamma \delta} \overline{\bar{C}}_{(k m)}^{i \beta \gamma \delta}+h^{-1} D_{(k \bullet)}^{(\bullet n)} \bar{C}_{(n m)}^{i \beta 33}, \\
\bar{C}_{(k m)}^{i 3 \gamma}=b_{\delta}^{\gamma} \bar{C}_{(k m)}^{i 33 \delta}+h^{-1} D_{(k \bullet)}^{(\bullet n)} C_{(n m)}^{i 3 \gamma 3} ; \quad \bar{C}_{(k m)}^{i 33}=-b_{\gamma \delta} \bar{C}_{(k m)}^{i 3 \gamma \delta}+h^{-1} D_{(k \bullet)}^{(\cdot n)} C_{(n m)}^{i 333} \cdot \\
\overline{\bar{C}}_{(k m)}^{i j k l}=\left(\overline{\bar{C}}^{i j k l} \bar{\mu} \mathrm{p}_{(k)}, \mathrm{p}_{(m)}\right) ; \quad \overline{\bar{C}}^{i \beta j \delta}=\bar{\mu} A_{\bullet \cdot}^{\beta \bullet} A_{\bullet \varepsilon}^{\delta \bullet} C^{i \gamma j \varepsilon} ; \bar{C}^{i \beta j 3}=\bar{\mu} A_{\bullet \gamma}^{\beta \cdot} C^{i \gamma j 3} ; \quad \bar{C}^{i 3 j \delta}=\bar{C}^{i 3 j \varepsilon}=\bar{\mu} A_{\bullet \varepsilon}^{\delta \cdot} C^{i \gamma j 3}, \\
\bar{\mu}=1+\bar{H}+\tilde{h}^{2} \bar{K}-2 \zeta(\bar{H}+\tilde{h} \bar{K})+\zeta^{2} \bar{K} ; \quad \bar{H}=h H, \quad \bar{K}=h^{2} K, \quad \tilde{h}=\bar{h} / h .
\end{gathered}
$$

Здесь $\overline{\bar{C}}_{(\mathrm{km})}^{i j p q}$ - физические константы теории N-го порядка, определенные в [45]. Постоянные $\overline{\bar{C}}^{i j k l}, \bar{C}^{i j k l}$ образуются при переходе от основного базиса пространственной системы координат $\xi^{\alpha}, \zeta$ к сопутствующему базису $\mathbf{r}_{\alpha}, \mathbf{n} ; \bar{H}, \bar{K}$ - безразмерные средняя и гауссова кривизны поверхности $S_{0} ; \tilde{h}\left(M_{0}\right)$ - отношение отклонения $\bar{h}$ базисной поверхности от срединной к толщине оболочки $h$. Уравнения (14), (17) с краевыми (15) и начальными условиями (16) формируют начально-краевую задачу трехмерной теории оболочек $\mathrm{N}$-го 
порядка [45-48]. Уравнения динамики (14) есть обобщенные уравнения Лагранжа II рода континуальной системы, определенной на двумерном многообразии $S_{0}[45,46]$.

\section{2. Формулировка расширенной теории оболочек $N$-го порядка}

Силовые краевые условия на $S_{ \pm} \subseteq S_{\sigma}$ имеют общий вид $\left.\boldsymbol{\sigma}\right|_{S \pm} \cdot \mathbf{n}^{ \pm}=\mathbf{q}^{ \pm}, \mathbf{n}^{ \pm}-$вектор единичной внешней нормали лицевой поверхности $S_{ \pm}$, компоненты которого определяются следующим образом:

$$
\begin{aligned}
\mathbf{n}^{ \pm}=\mathbf{N}^{ \pm} /\left|\mathbf{N}^{ \pm}\right| ; \quad \mathbf{N}^{ \pm} & =\mathbf{R}_{1}^{ \pm} \times \mathbf{R}_{2}^{ \pm}, \quad \mathbf{R}_{\beta}^{ \pm}=\left.\partial_{\beta} \mathbf{R}\right|_{\zeta= \pm 1} ; \quad \Rightarrow \mathbf{N}^{ \pm}=\mu_{ \pm} \sqrt{a} \mathbf{n}, \quad\left|\mathbf{N}^{ \pm}\right|=\mu_{ \pm} \sqrt{a}, \\
& \Rightarrow \mathbf{n}^{ \pm}=v_{ \pm}^{-1} \mathbf{n} ; \quad \mu_{ \pm}=1-h_{ \pm} b_{\lambda}^{\lambda}+h_{ \pm}^{2} \operatorname{det}\left(b_{\beta}^{\alpha}\right) .
\end{aligned}
$$

Главный вектор внешних сил на $S_{ \pm}$записывается так: $\mathbf{q}^{ \pm}=\left(\mu_{ \pm} v_{ \pm}\right)^{-1}\left(q_{ \pm}^{\alpha} \mathbf{r}_{\alpha}+q_{ \pm}^{3} \mathbf{n}\right)$, при этом силовые краевые условия на лицевых поверхностях приводятся к виду

$$
\left.s^{i 3}\right|_{S \pm}=\bar{q}_{ \pm}^{i}, i=\overline{1,3}, \beta=\overline{1,2} .
$$

При учете определяющих уравнений для $s_{(k)}^{i j}(17)$, полученных в $[45,46]$ для случая анизотропной упругой среды, краевые условия (18) переходят в уравнения связей для переменных поля І рода $u_{j}^{(k)}$, определенные на $S_{0}$ :

$$
\begin{gathered}
C_{(k) \pm}^{i j \delta}\left(\bar{\nabla}_{\delta} u_{j}^{(k)}+H_{\delta(m \bullet)}^{(\bullet k)} u_{j}^{(m)}\right)+C_{(k) \pm}^{i j 3} D_{(m \bullet)}^{(\bullet k)} u_{j}^{(m)}-C_{(k) \pm}^{i \gamma \delta} b_{\gamma \delta} u_{3}^{(k)}+C_{(k) \pm}^{i 3 \delta} b_{\delta}^{\gamma} u_{\gamma}^{(k)}-\bar{q}_{ \pm}^{i}=0 \\
C_{(k) \pm}^{i j \delta}=\left(\bar{C}_{(k m)}^{i 3 j \delta}-h_{\beta}^{ \pm} \overline{\bar{C}}_{(k m)}^{i \beta j \delta}\right) \mathrm{p}^{(m)}( \pm 1) ; \quad C_{(k) \pm}^{i j 3}=\left(C_{(k m)}^{i 3 j 3}-h_{\beta}^{ \pm} \bar{C}_{(k m)}^{i \beta j 3}\right) \mathrm{p}^{(m)}( \pm 1) .
\end{gathered}
$$

Учет краевых условий (18) на лицевых поверхностях $S_{ \pm}$в форме связей (19) преобразует экстремальную задачу для функционала (11), (12) в экстремальную задачу с ограничениями, решаемую методом множителей Лагранжа [44]. Начально-краевая задачи теории оболочек N-го порядка, следующая из (11), (12) и (19), имеет вид

$$
\begin{gathered}
\rho_{(k)}^{(m)} \ddot{u}_{(m)}^{\alpha}=\bar{\nabla}_{\beta} \tilde{s}_{(k)}^{\alpha \beta}-b_{\beta}^{\alpha} \tilde{s}_{(k)}^{3 \beta}-D_{(k \cdot)}^{(\cdot m)} \tilde{s}_{(m)}^{\alpha 3}+P_{(k)}^{\alpha}, \\
\rho_{(k)}^{(m)} \ddot{u}_{(m)}^{3}=\bar{\nabla}_{\beta} \tilde{s}_{(k)}^{3 \beta}+b_{\alpha \beta} \tilde{s}_{(k)}^{\alpha \beta}-D_{(k \cdot)}^{(\cdot m)} \tilde{s}_{(m)}^{33}+P_{(k)}^{3}, \\
\left.\left(\tilde{s}_{(k)}^{i \beta} v_{\beta}-q_{B(k)}^{i}\right) \delta u_{i}^{(k)}\right|_{M_{0} \in \Gamma}=0 ; \quad i=1,2,3 ; \quad \beta=1,2, \\
{\left[\tilde{s}_{(k)}^{i 3}-\lambda_{j}^{ \pm} C_{(k) \pm}^{j i 3}\right] p^{(k)}( \pm 1)-\bar{q}_{ \pm}^{i}=0,} \\
\tilde{s}_{(k)}^{i \beta}=\overline{\bar{C}}_{(k m)}^{i \beta j \delta} \bar{\nabla}_{\delta} u_{j}^{(m)}+\bar{C}_{(k m)}^{i \beta j} u_{j}^{(m)}+\lambda_{j}^{+} C_{(k)+}^{j i \beta}+\lambda_{j}^{-} C_{(k)-}^{j i \beta}, \\
\tilde{s}_{(k)}^{i 3}=\bar{C}_{(k m)}^{i 3 j \delta} \bar{\nabla}_{\delta} u_{j}^{(m)}+C_{(k m)}^{i 3 j} u_{j}^{(m)}+\lambda_{j}^{+} C_{(k)+}^{j i 3}+\lambda_{j}^{-} C_{(k)-}^{j i 3}, \\
\left.u_{(k)}^{i}\right|_{t=t_{0}}=U_{(k)}^{i} ;\left.\quad \dot{u}_{(k)}^{i}\right|_{t=t_{0}}=V_{(k)}^{i} .
\end{gathered}
$$


Здесь введены соответствующие лицевым поверхностям оболочки $S_{+}$и $S_{-}$векторы множителей Лагранжа $\lambda^{ \pm}=\lambda_{\alpha}^{ \pm} \mathbf{r}^{\alpha}+\lambda_{3}^{ \pm} \mathbf{n}$, а также следующие обозначения обобщенных физических постоянных:

$$
C_{(k) \pm}^{i j 3}=C_{(k m)}^{i 3 j 3} p^{(m)}( \pm 1)
$$

\section{3. Уравнения движения плоского изотропного упругого слоя}

В случае плоского однородного изотропного упругого слоя с учетом

$$
\begin{gathered}
b_{\alpha}^{\beta}=b_{\alpha \beta}=0 \Rightarrow A_{\alpha \cdot}^{\bullet \beta}=A_{\cdot \alpha}^{\beta \cdot} \equiv \delta_{\alpha}^{\beta} \Rightarrow \tilde{s}_{(k)}^{i j}=\tilde{\sigma}_{(k)}^{i j}, \quad s_{(k)}^{i j}=\sigma_{(k)}^{i j}, \\
\rho_{(k)}^{(m)}=\rho \delta_{(k)}^{(m)} ; \quad \overline{\bar{C}}^{i j p q} \equiv \bar{C}^{i j p q} \equiv C^{i j p q}=\lambda g^{i j} g^{p q}+\mu\left(g^{i p} g^{j q}+g^{i q} g^{j p}\right) ; \quad C_{(k m)}^{i j p q}=C^{i j p q} G_{(k m)}, \\
C_{(k) \pm}^{j i 3}=C^{j 3 i 3} \mathrm{p}_{(k)}( \pm 1)=\mathrm{p}_{(k)}( \pm 1) \begin{cases}\mu a^{\alpha \beta}, & i=\alpha, j=\beta, \\
\lambda+2 \mu, & i=3, \mathrm{j}=3, \\
0, & i=\alpha, j=3, i \rightleftarrows j\end{cases}
\end{gathered}
$$

уравнения связей преобразуются к виду

$$
\begin{aligned}
& \tilde{\sigma}_{(k)}^{\alpha 3} p^{(k)}( \pm 1)-\lambda_{\beta}^{ \pm} \mu a^{\alpha \beta} p_{N}-\bar{q}_{ \pm}^{\alpha}=0 ; \tilde{\sigma}_{(k)}^{33} p^{(k)}( \pm 1)-\lambda_{3}^{ \pm}(\lambda+2 \mu) p_{N}-\bar{q}_{ \pm}^{3}=0 \\
& p_{N}=\mathrm{p}_{(k)}( \pm 1) \mathrm{p}^{(k)}( \pm 1)=G_{(k m)} \mathrm{p}^{(k)}( \pm 1) \mathrm{p}^{(m)}( \pm 1)=G^{(k m)} \mathrm{p}_{(k)}( \pm 1) \mathrm{p}_{(m)}( \pm 1)
\end{aligned}
$$

Для базиса, образованного полиномами Лежандра, $p_{N}$ запишется так:

$$
p_{N}=\mathrm{p}_{(k)}( \pm 1) \mathrm{p}^{(k)}( \pm 1)=\sum_{k=0}^{N}\left\|\mathrm{p}^{(k)}\right\|^{2} \Leftarrow \mathrm{p}_{(k)}( \pm 1)=( \pm 1)^{k}, G^{(k m)}=\operatorname{diag}\left(\left\|\mathrm{p}^{(k)}\right\|^{2}\right) .
$$

В силу $\left|a_{\alpha \beta}\right| \neq 0$ уравнения связей разрешимы относительно множителей $\lambda_{j}^{ \pm}$:

$$
\lambda_{3}^{ \pm}=(\lambda+2 \mu)^{-1} p_{N}^{-1}\left[\tilde{\sigma}_{(k)}^{33} \mathrm{p}^{(k)}( \pm 1)-\bar{q}_{3}^{ \pm}\right] ; \quad \lambda_{\beta}^{ \pm}=\mu^{-1} p_{N}^{-1} a_{\alpha \beta}\left[\tilde{\sigma}_{(k)}^{\alpha 3} \mathrm{p}^{(k)}( \pm 1)-\bar{q}_{ \pm}^{\alpha}\right] .
$$

Определяющие уравнения будут иметь следующий вид:

$$
\begin{gathered}
\tilde{\sigma}_{(k)}^{\alpha \beta}=\sigma_{(k)}^{\alpha \beta}+\lambda a^{\alpha \beta}\left(\lambda_{3}^{+} \mathrm{p}_{(k)}(1)+\lambda_{3}^{-} \mathrm{p}_{(k)}(-1)\right), \\
\tilde{\sigma}_{(k)}^{\alpha 3}=\sigma_{(k)}^{\alpha 3}+\mu a^{\alpha \gamma}\left(\lambda_{\gamma}^{+} \mathrm{p}_{(k)}(1)+\lambda_{\gamma}^{-} \mathrm{p}_{(k)}(-1)\right), \\
\tilde{\sigma}_{(k)}^{33}=\sigma_{(k)}^{33}+(\lambda+2 \mu)\left(\lambda_{3}^{+} \mathrm{p}_{(k)}(1)+\lambda_{3}^{-} \mathrm{p}_{(k)}(-1)\right) .
\end{gathered}
$$

Подстановка $\lambda_{j}^{ \pm}$в определяющие уравнения и разрешение их относительно $\tilde{\sigma}_{(k)}^{i j}$ приводит к уравнениям, связывающим обобщенные усилия $\tilde{\sigma}_{(k)}^{i j}$ и $\sigma_{(k)}^{i j}$ :

$$
\tilde{\sigma}_{(k)}^{\alpha \beta}=\left[\sigma_{(k)}^{\alpha \beta}-\frac{\lambda}{\lambda+2 \mu} a^{\alpha \beta} \sigma_{(k)}^{33}\right]+\frac{\lambda}{\lambda+2 \mu} a^{\alpha \beta} R_{(k)}^{(m)}\left[\sigma_{(m)}^{33}-p_{N}^{-2}\left(\bar{q}_{+}^{3} \mathrm{p}_{(m)}^{+}+\bar{q}_{-}^{3} \mathrm{p}_{(m)}^{-}\right)\right],
$$




$$
\begin{gathered}
\tilde{\sigma}_{(k)}^{\alpha 3}=\sigma_{(k)}^{\alpha 3}-R_{(k)}^{(n)} P_{(n)}^{(m)}\left[\sigma_{(m)}^{\alpha 3}-p_{N}^{-2}\left(\bar{q}_{+}^{\alpha} \mathrm{p}_{(m)}^{+}+\bar{q}_{-}^{\alpha} \mathrm{p}_{(m)}^{-}\right)\right], \\
\tilde{\sigma}_{(k)}^{33}=R_{(k)}^{(m)} \sigma_{(m)}^{33}-R_{(k)}^{(n)} p_{N}^{-2}\left(\bar{q}_{+}^{3} \mathrm{p}_{(m)}^{+}+\bar{q}_{-}^{3} \mathrm{p}_{(m)}^{-}\right) . \\
P_{(k)}^{(m)}=p_{N}^{-1}\left(\mathrm{p}_{(k)}^{+} \mathrm{p}_{+}^{(m)}+\mathrm{p}_{(k)}^{-} \mathrm{p}_{-}^{(m)}\right) ; \quad R_{(k)}^{(n)} \bar{P}_{(n)}^{(m)}=\delta_{(k)}^{(m)} ; \quad \bar{P}_{(k)}^{(m)}=\delta_{(k)}^{(m)}-P_{(k)}^{(m)} . \\
p_{N}^{2}=\mathrm{p}_{(k)}^{ \pm} \mathrm{p}_{ \pm}^{(k)}>0 .
\end{gathered}
$$

Начально-краевая задача расширенной теории пластин $N$-го порядка примет вид

$$
\begin{gathered}
\rho \ddot{u}_{(k)}^{i}=\bar{\nabla}_{\beta} \tilde{\sigma}_{(k)}^{i \beta}-D_{(k \cdot)}^{(\cdot m)} \tilde{\sigma}_{(m)}^{i 3}+P_{(k)}^{i}, \\
\left.\left(\tilde{\sigma}_{(k)}^{i \beta} v_{\beta}-q_{B(k)}^{i}\right) \delta u_{i}^{(k)}\right|_{M_{0} \in \Gamma}=0 ; \quad i=1,2,3 ; \quad \beta=1,2, \\
\left.u_{(k)}^{i}\right|_{t=t_{0}}=U_{(k)}^{i} ;\left.\quad \dot{u}_{(k)}^{i}\right|_{t=t_{0}}=V_{(k)}^{i} .
\end{gathered}
$$

\section{4. Спектральная задача для плоского упругого слоя}

Рассмотрим задачу о распространении второй моды продольной волны в плоском упругом слое плотностью $\rho$, с параметрами Ламе $\lambda, \mu$, толщиной $2 h$ и со срединной плоскостью $x_{2}=0$. Нормальная волна распространяется в направлении оси $O x_{1}$ прямоугольной декартовой системы координат $O x_{1} x_{2} x_{3}$. На поверхностях слоя $x_{2}= \pm h$ поставлены однородные краевые условия $\sigma_{\alpha 2}\left(x_{1}, \pm h\right)=0$.

Аналогично [53-57] введем безразмерные переменные

$$
\xi=x_{1} h^{-1} ; \quad \tau=t c_{1} h^{-1} ; \quad \tilde{u}_{\alpha}^{(k)}=u_{\alpha}^{(k)} h^{-1}, \quad \beta=c_{2} / c_{1}=(1-2 v) /(2-2 v),
$$

где $c_{1}=\sqrt{(\lambda+2 \mu) \rho^{-1}}, c_{2}=\sqrt{\mu \rho^{-1}}-$ скорости распространения волн дилатации и сдвига; $v$ - коэффициент Пуассона.

Однородные уравнения движения в безразмерных переменных, соответствующие расширенной теории N-го порядка [45-48], следуют из (14)-(17) и приводятся к матричной форме записи

$$
\begin{gathered}
\partial_{\tau}^{2} \mathbf{u}=\left(\mathbf{C}_{2} \partial_{\xi}^{2}+\mathbf{C}_{1} \partial_{\xi}+\mathbf{C}_{0}\right) \mathbf{u}, \\
\mathbf{u}=\left[\begin{array}{llll}
u_{1}^{(0)} \ldots & u_{1}^{(N)} & u_{2}^{(0)} \ldots & u_{2}^{(N)}
\end{array}\right] ; \quad \mathbf{C}_{K}=\mathbf{A}_{K}+\mathbf{B}_{K}, \quad K=0,1,2 .
\end{gathered}
$$

Операторы $D_{(k \bullet)}^{(\cdot m)}(25)$ и $\bar{D}_{(\cdot m)}^{(k \cdot)}$ соответствуют базису, образованному полиномами Лежандра $\mathrm{p}_{(k)}(\zeta), \zeta=x_{2} / h \in[-1,1]$ в соответствии с [37-43, 45-49]:

$$
D_{(\bullet m)}^{(k \bullet)}=(2 m+1) \delta_{(k)}^{(m+2 l+1)}, l<\left[\frac{1}{2}(N-m-1)\right] ; \quad \bar{D}_{(\cdot m)}^{(k \bullet)}=(2 m+3) \delta_{(k)}^{(m-2 l-1)}, l<\left[\frac{1}{2}(m-1)\right] .
$$

Матрицы линейных операторов $D_{(m \bullet)}^{(\cdot k)}, \bar{D}_{(\cdot m)}^{(k \bullet)}$ имеют размерность $(N+1) \times(N+1)$. 
Вектор перемещения, соответствующий нормальной волне, может быть представлен в виде [53-57]

$$
\mathbf{u}^{(k)}=\mathbf{U}^{(k)} \exp \left[i\left(k x_{1}-\Omega t\right)\right]=\mathbf{U}^{(k)} \exp [i(\kappa \xi-\omega \tau)]
$$

где $\mathbf{U}^{(k)}$ - амплитуда; $\omega=\Omega h / c_{2}$ - безразмерная фазовая частота; $\kappa=k h-$ безразмерное волновое число. С учетом (42) уравнения движения расширенной теории $N$-го порядка (...) приводятся к $2 N+2$ линейному уравнению:

$$
\begin{aligned}
& \left(\mathbf{C}_{2} \kappa^{2}+i \mathbf{C}_{1} \kappa+\mathbf{C}_{0}-\omega^{2} \mathbf{I}\right) \mathbf{U}=\mathbf{0}, \\
& \mathbf{U}=\left[\begin{array}{llll}
U_{1}^{(0)} \ldots & U_{1}^{(N)} & U_{2}^{(0)} \ldots & U_{2}^{(N)}
\end{array}\right], \quad \mathbf{C}_{J}=\mathbf{A}_{J}+\mathbf{B}_{J}, \quad J=1,2 ; \\
& \mathbf{A}_{2}=\left(\begin{array}{cc}
4 \beta^{2}\left(1-2 \beta^{2}\right) \delta_{(k)}^{(m)} & 0 \\
0 & \beta^{2} \delta_{(k)}^{(m)}
\end{array}\right) \\
& \mathbf{A}_{1}=\left(\begin{array}{cc}
0 & \beta^{2} D_{(\bullet m)}^{(k \cdot)} \\
-\beta^{2} \bar{D}_{(\bullet m)}^{(k \cdot)} & 0
\end{array}\right) \\
& \mathbf{A}_{0}=\left(\begin{array}{cc}
\beta^{2} D_{(\bullet n)}^{(k \cdot)} \bar{D}_{(\cdot m)}^{(n \bullet)} & 0 \\
0 & 0
\end{array}\right) \\
& \mathbf{B}_{2}=\left(\begin{array}{cc}
4 \beta^{2}\left(1-2 \beta^{2}\right) R_{(k)}^{(m)} & 0 \\
0 & \beta^{2} R_{(k)}^{(m)}
\end{array}\right), \\
& \mathbf{B}_{1}=\left(\begin{array}{cc}
0 & \beta^{2} D_{(\cdot l)}^{(k \bullet)} R_{(n)}^{(l)} P_{(m)}^{(n)}-2\left(1-2 \beta^{2}\right) R_{(n)}^{(k)} \bar{D}_{(\cdot m)}^{(n \bullet)} \\
2\left(1-2 \beta^{2}\right) D_{(\cdot n)}^{(k \bullet)} R_{(m)}^{(n)}-\beta^{2} R_{(l)}^{(k)} P_{(n)}^{(l)} \bar{D}_{(\bullet m)}^{(n \bullet)} & 0
\end{array}\right), \\
& \mathbf{B}_{0}=\left(\begin{array}{cc}
\beta^{2} D_{(\cdot l)}^{(k \bullet)} R_{(n)}^{(l)} \bar{D}_{(\cdot m)}^{(n \bullet)} & 0 \\
0 & \bar{D}_{(\bullet l)}^{(k \bullet)} R_{(n)}^{(l)} D_{(\bullet m)}^{(n \bullet)}
\end{array}\right),
\end{aligned}
$$

Здесь I - единичная; 0 - нулевая матрицы размерности $(2 N+2) \times(2 N+2)$. Операторы $\mathbf{A}_{K}$ соответствуют плоскому напряженному состоянию, операторы $\mathbf{B}_{K}$ учитывают влияние связей. Из уравнений движения (43) следует спектральная задача [53-57]

$$
\left|\left(\mathbf{C}_{0}+i \kappa \mathbf{C}_{1}+\kappa^{2} \mathbf{C}_{2}\right)-\omega^{2} \mathbf{I}\right|=0
$$

Собственные значения оператора $(43) \omega_{n}(\kappa) \in R_{+}$определяют на плоскости $(\kappa, \omega)$ дисперсионные кривые; $\kappa \in \mathrm{R}$ соответствуют распространяющимся модам [58]. Собственные векторы $\mathbf{U}^{n}(\kappa), n \in[1,2 N+2] \cap \mathbb{Z}$ задают формы мод: 


$$
u_{\alpha}^{n}(\zeta)=U_{\alpha}^{(k) n} \mathrm{p}_{k}(\zeta), \quad \alpha=1,2, \quad k=0 \ldots N, \quad n=1 \ldots N+1 .
$$

Система уравнений (43) распадается на независимые подсистемы для симметричных и антисимметричных относительно плоскости $\zeta=0$ волн, при этом продольным волнам соответствуют индексы

$$
k, m=\{2 n, N+2 n+2\} ; \quad n \in\left[0,\left[\frac{1}{2}(N+1)\right]\right] \cup \mathbb{Z} .
$$

\section{5. Анализ описания формы второй распространяющейся продольной моды нормальных волн теориями различного порядка}

Рассмотрим далее вторую распространяющуюся моду продольных нормальных волн: $\omega_{2}(\kappa), \mathbf{U}^{2}(\kappa), \kappa \in\left[0, \kappa^{*}\right][48,49,57]$. На рис. 1 приведены дисперсионные кривые для фазовой частоты $\omega_{2}(\kappa)$ и групповой скорости $c_{g 2}(\kappa)$, соответствующие второй распространяющейся моде, построенные на основе результатов $[48,49]$ и точного решения задачи Рэлея-Лэмба [58].

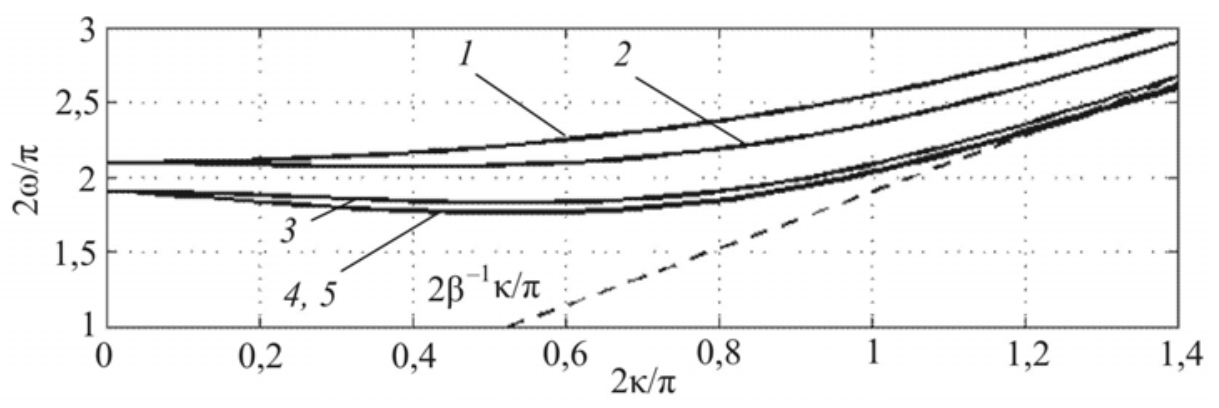

$a$

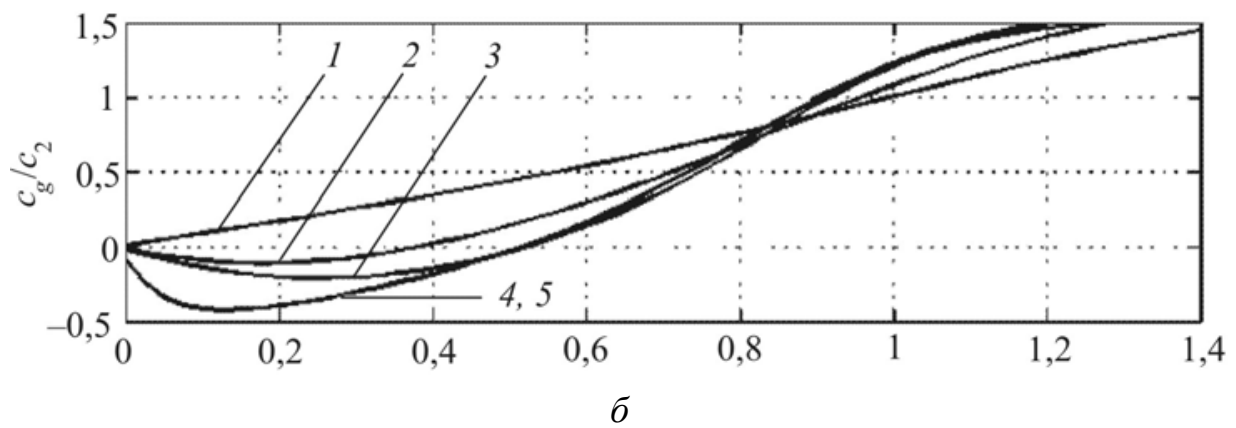

Рис. 1. Фазовая частота $\omega(a)$ и групповая скорость $c_{g}($ б) второй распространяющейся моды продольной волны в упругом изотропном слое. Теории $1,2 \ldots 5$-го порядков $[48,49]$

Достаточной для практических приложений $[59,60]$ точности вычисления групповой скорости $c_{g}$ удается достичь при $N=4$. При $N \leq 3$ зависимость групповой скорости в области «обратной волны» качественно отличается от точного решения [58].

Рассмотрим далее эволюцию формы второй распространяющейся моды при $\kappa^{*}<1,4$. Согласно (51) соотношения

$$
u_{1}^{2}(\kappa, \zeta)=U_{1}^{(k) 2}(\kappa) \mathrm{p}_{(k)}(\zeta), \quad k=0,2 \ldots N^{\prime}, \quad N^{\prime} \leq N
$$


описывают зависимость продольного перемещения от нормальной координаты $\zeta$ и волнового числа $\kappa$; аналогичное соотношение описывает поперечное перемещение $u_{2}$ :

$$
u_{2}^{2}(\kappa, \zeta)=U_{2}^{(k) 2}(\kappa) \mathrm{p}_{(k)}(\zeta), \quad k=1,3 \ldots N^{\prime \prime}, \quad N^{\prime \prime} \leq N,
$$

где выбор индексов $(k)$ соответствует базису, образованному полиномами Лежандра при симметрии перемещения в продольной волне: $u_{1}^{n}(\zeta)=u_{1}^{n}(-\zeta) ; u_{2}^{n}(\zeta)=-u_{2}^{n}(-\zeta)$.

\section{6. Кинематика второй распространяющейся моды}

На рис. 2 приведена зависимость $u_{1}^{2}(\zeta, \kappa), \kappa \in[0,1,5], \zeta \in[-1,1], N=5$.

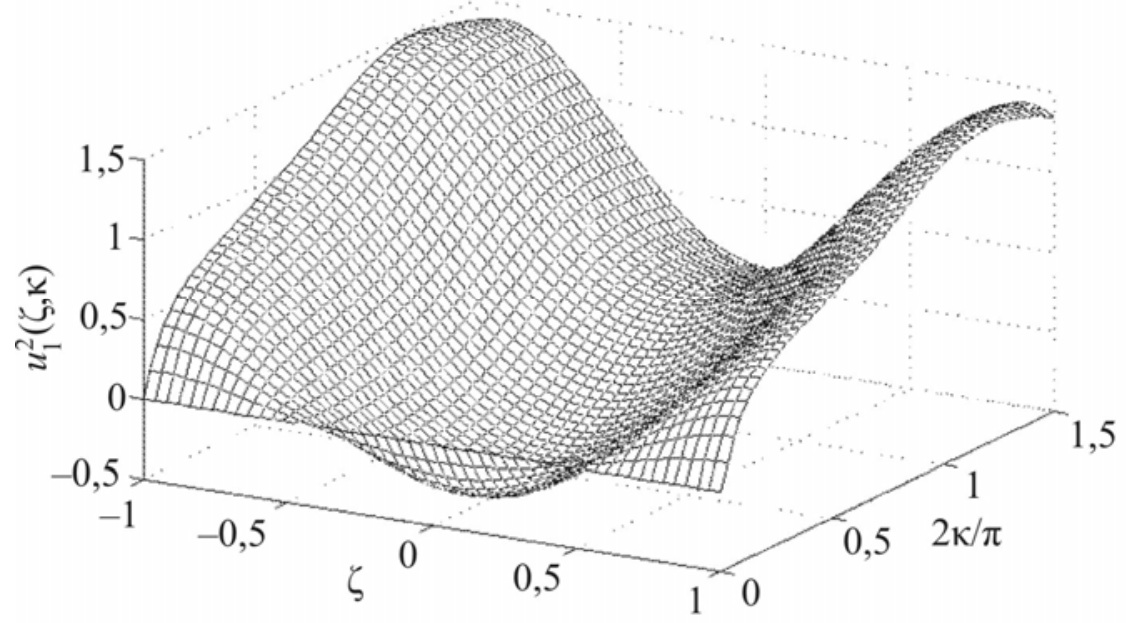

Рис. 2. Эволюция формы второй распространяющейся моды продольной нормальной волны в слое в области эффекта «обратной волны».

Продольное перемещение $u_{1}^{2}(\kappa, \zeta)(52)$. Теория 5-го порядка

На рис. 3 показаны зависимости безразмерного перемещения $u_{1}^{2}(\zeta, \kappa)$, определяемого следующим соотношением [57]:

$$
u_{\alpha}^{2}(\zeta, \kappa)=u_{\alpha}^{2} / \max _{\zeta \in[-1,1]}\left|u_{\alpha}^{2}(\zeta, \kappa)\right|,
$$

от нормальной координаты $\zeta$ при характерных значениях волнового числа $\kappa$, соответствующих $c_{g}(\kappa)=\min$ и $c_{g}(\kappa)=0, N=2 \ldots 6$. Кривые на рис. 3 соответствуют точному решению, полученному в [58]. Как при минимальной отрицательной скорости $c_{g}^{\min }$, так и при $c_{g}=0$ в сечении слоя существуют подобласти с противоположными знаками амплитуд продольного перемещения:

$$
c_{g}=c_{g}^{\min }:\left.\quad \zeta\right|_{u_{1}^{2}<0} \in(\approx[-0,5,0,5]) ; \quad c_{g}=0:\left.\quad \zeta\right|_{u_{1}^{2}<0} \in(\approx[-0,48,0,48]) .
$$

Распределения по толщине слоя безразмерного продольного перемещения $u_{1}^{2}(\zeta, \kappa)$ в точке дисперсионной кривой, соответствующей $c_{g}^{\min }$, описываемые теориями порядка 
$N \geq 4$, практически совпадают [57]. Зависимости $u_{1}^{2}(\zeta, \kappa)$, соответствующие $N=2,3$, качественно отличаются, представляя собой параболическую аппроксимацию перемещения. Аналогичный вывод следует из анализа зависимости $u_{1}^{2}(\zeta, \kappa)$ при $\kappa: c_{g}=0$. Следует отметить, что в точке $c_{g}^{\min }$ границы подобласти $u_{1}^{2}<0$, вычисленные на базе теорий $2 \ldots 6$ порядков, практически совпадают, несмотря на качественное различие кривых. В точке $\kappa: c_{g}=0$ границы подобласти $u_{1}^{2}<0$, определяемые теориями 2, 3-го и высших порядков, различаются.

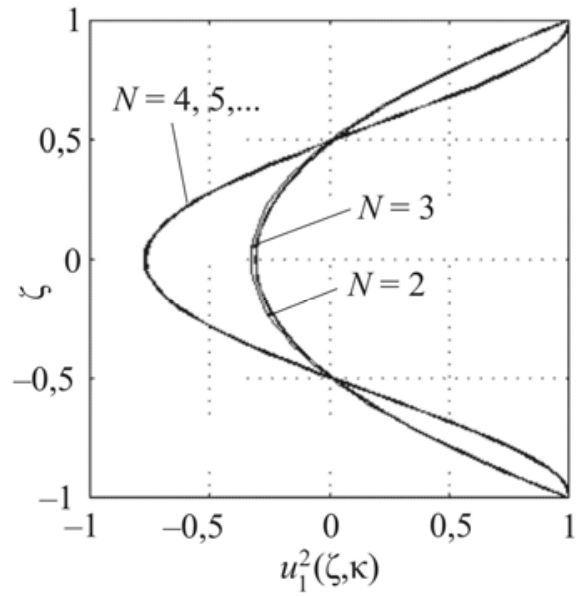

$a$

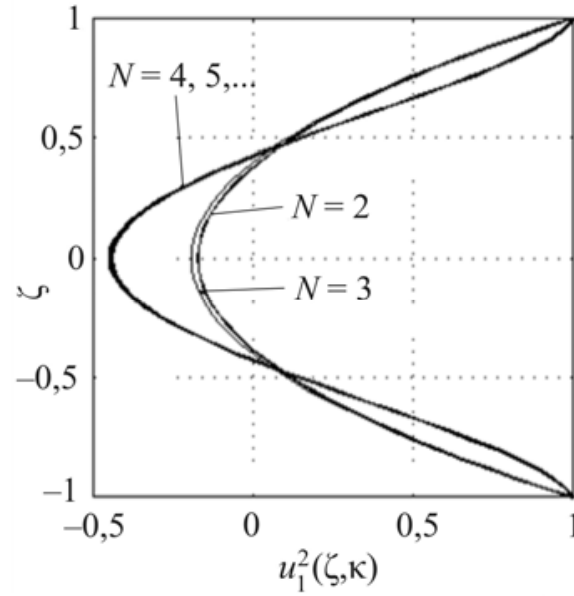

$\sigma$

Рис. 3. Распределение по толщине слоя безразмерного продольного перемещения:

(a) $c_{g}=\min ;$ (б) $c_{g}=0$. Теории 2, 3, 4, 5-го порядков

На рис. 4 показана эволюция формы поперечного перемещения $u_{2}^{2}(\zeta, \kappa)$ во второй распространяющейся моде, при $\kappa \in[0,1,5], \zeta \in[-1,1], N=5$.

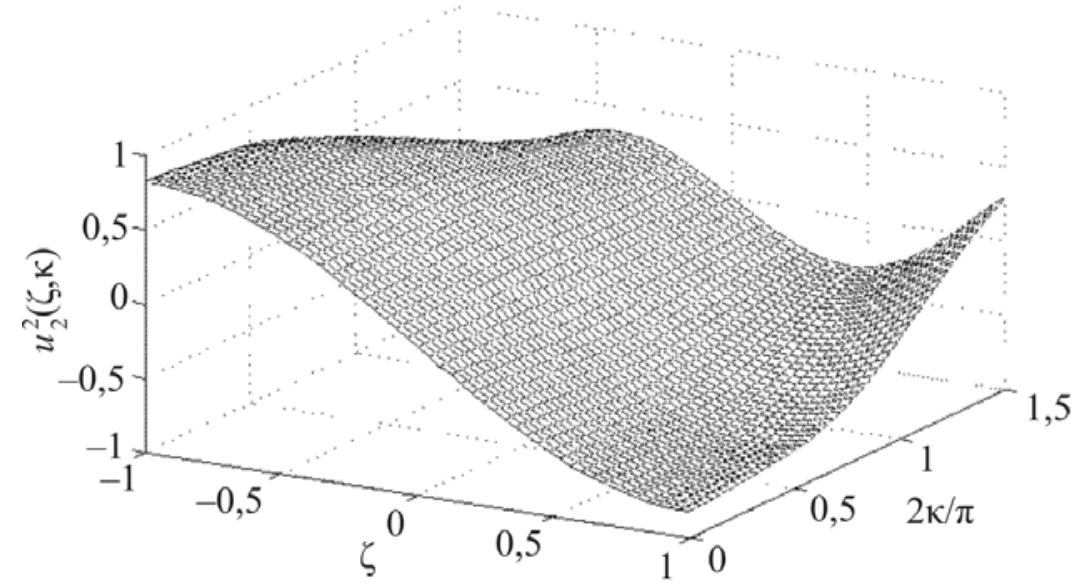

Рис. 4. Эволюция формы второй распространяющейся моды продольной нормальной волны в слое в области эффекта «обратной волны».

Поперечное перемещение $u_{2}^{2}(\kappa, \zeta)(53)$. Теория 5-го порядка 
На рис. 5 приведены зависимости безразмерного перемещения $\mathrm{u}_{2}^{2}(\zeta, \kappa)$, определяемого (54), от координаты $\zeta$ при значениях волнового числа $\kappa: c_{g}(\kappa)=\min$ и $\kappa:$ $c_{g}(\kappa)=0$. Аналогично предыдущему случаю $N=2 \ldots 6$.

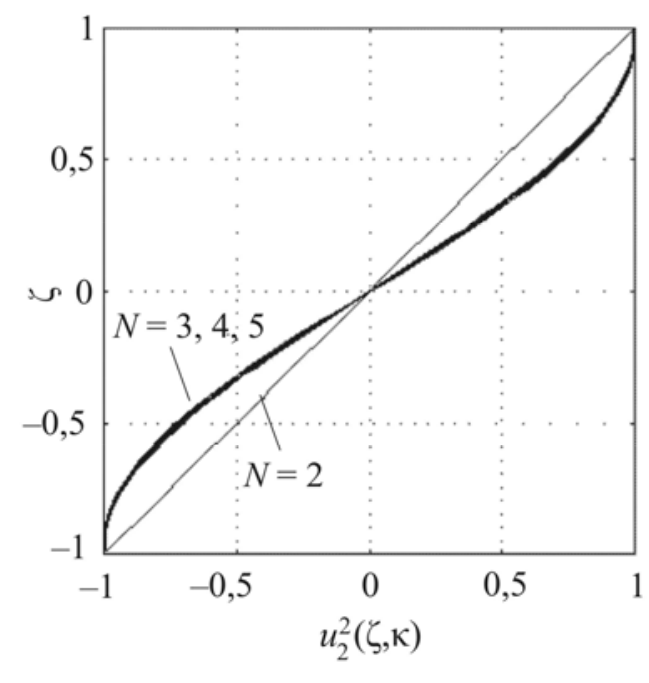

$a$

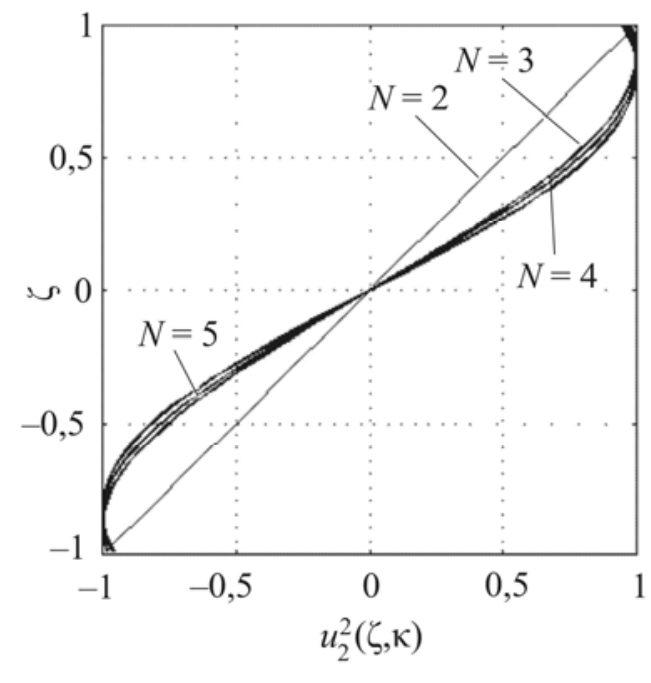

$\sigma$

Рис. 5. Распределение по толщине слоя безразмерного поперечного перемещения:

(a) $c_{g}=\min ;\left(\right.$ ) $c_{g}=0$. Теории 2, 3, 4, 5-го порядков

Распределение поперечного безразмерного перемещения $u_{2}^{2}(\zeta, \kappa)$ по толщине слоя, вычисленное на базе теории второго порядка (см. рис. 5), соответствует линейной аппроксимации и качественно отличается от зависимости $u_{2}^{2}$, полученной на основе теорий $3,4,5$-го и высших порядков. При этом в точке $\kappa: c_{g}^{\min }$ кривые для всех порядков теории $N \geq 3$ совпадают, в точке $\kappa: c_{g}=0$ различие между кривыми, соответствующими $3,4,5$-му порядкам, становится заметным.

По мере повышения порядка кривизна кривых в областях $|\zeta| \in[0,5,1]$ увеличивается. При $N>5$ кривые совпадают.

На рис. 6, 7 приведены распределения по толщине слоя продольного $(a)$ и поперечного (б) перемещений при дальнейшем увеличении значения волнового числа. Рис. 6 соответствует $2 \kappa / \pi=1$, рис. 7 - значению $2 \kappa / \pi=1,5$. Как следует из рис. 1 , при таких величинах волнового числа знаки фазовой и групповой скоростей совпадают.

Анализируя приведенные на рис. 6, 7 результаты, можно утверждать, что на ближайшем к области проявления эффекта «обратной волны» отрезке $\kappa \leq 1$ точность теории 4-5-го порядков достаточна для описания поперечного перемещения $u_{2}^{2}$.

Для достаточной точности аппроксимации продольного перемещения предпочтительна теория 5-го порядка. При $2 \kappa / \pi \approx 1,5$ погрешность теории 5-го порядка становится заметной. 


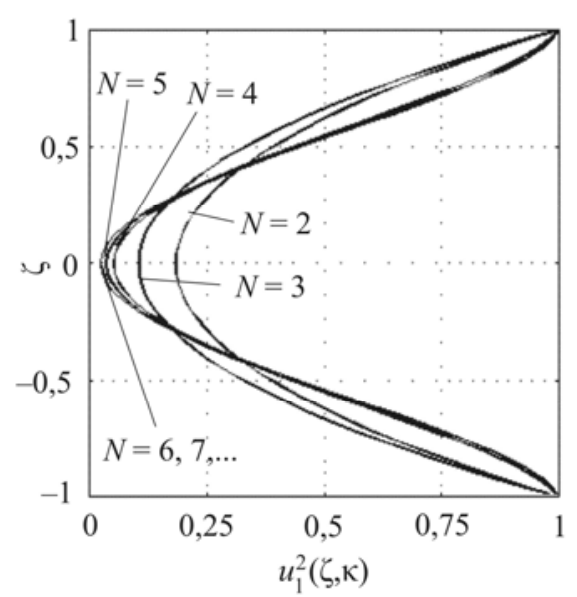

$a$

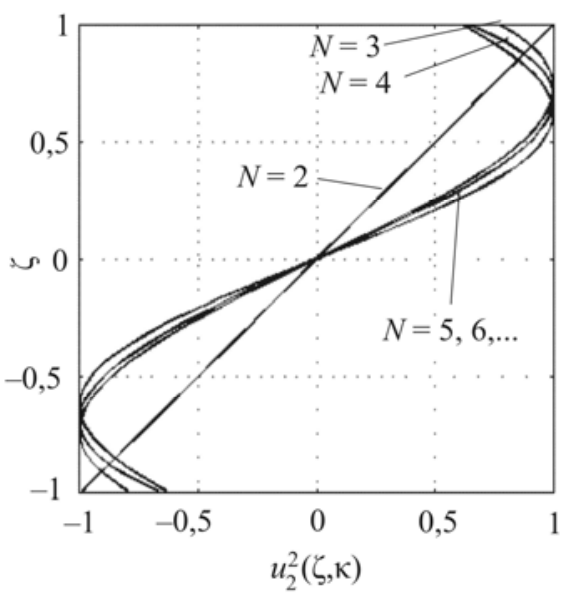

$\sigma$

Рис. 6. Распределение по толщине слоя компонентов безразмерного перемещения при значении волнового числа $2 \kappa / \pi=1$.

Теории 2, 3, 4, 5, 6, 7-го порядков

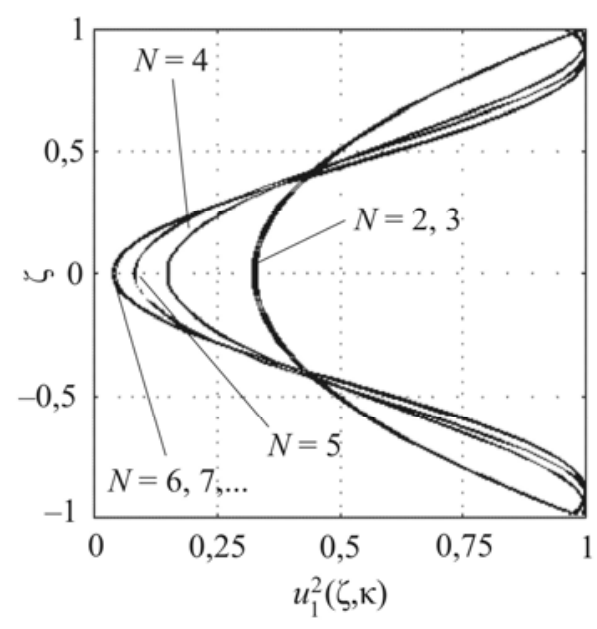

$a$

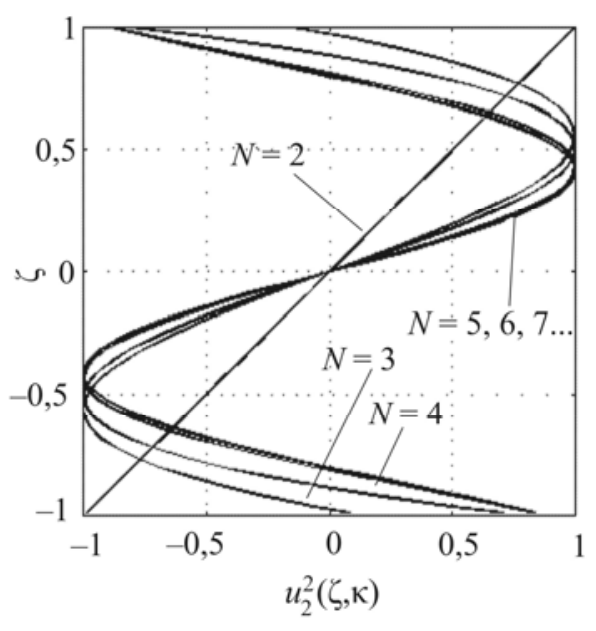

$\sigma$

Рис. 7. Распределение по толщине слоя компонентов безразмерного перемещения при значении волнового числа $2 \kappa / \pi=1,5$.

Теории 2, 3, 4, 5, 6, 7-го порядков

\section{Заключение}

Получена формулировка расширенной теории N-го порядка нетонких анизотропных оболочек, учитывающая силовые краевые условия на лицевых поверхностях, перенесенных на базовую поверхность оболочки, в форме неголономных уравнений связей. Для плоского упругого слоя получена формулировка уравнений движения в переменных поля первого рода и сформулирована соответствующая спектральная задача. Проведенный анализ аппроксимации формы второй распространяющейся моды нормальных волн в упругом слое на базе теории изотропных пластин N-го порядка И.Н. Векуа - А.А. Амосова [31-33, 37-43] в варианте [45-49], при использовании в качестве базисных функций полиномов Лежандра, соответствующих традиционной формулировке «трехмерных» теорий оболочек [19-22, 31-36], позволяет сделать следующие основные выводы. 
1. Для обнаружения эффекта «обратной волны» в плоском упругом слое, т.е. для получения в результате решения соответствующей спектральной задачи на некотором отрезке $\kappa \in[0, \kappa *]$ отрицательных значений групповых скоростей $c_{g}<0$ второй распространяющейся продольной моды, минимально необходимой является теория 2-го порядка [48]. В то же время теория 2-го порядка приводит к существенному занижению к*, а также получению завышенного значения $c_{g}^{\min }$. Продольное перемещение при этом описывается параболической, поперечное - линейной аппроксимацией. Теория 2-го порядка не может быть использована при решении задач, связанных с учетом явления «обратной волны».

2. Теория 3-го порядка за счет дополнительной степени свободы в трансверсальном направлении, обеспечивающей кубическую аппроксимацию поперечного перемещения и, следовательно, лучшее приближение в области «толщинного резонанса» [53] при малых значениях волнового числа, позволяет получить практически точное значение $2 \kappa * / \pi \approx 0,51$ границы области проявления «эффекта обратной волны», однако приводит к завышению $c_{g}^{\min }$ и смещению точки минимума групповой скорости в сторону больших волновых чисел. Продольное перемещение в рамках теории 3-го порядка описывается параболической аппроксимацией и близко к аппроксимации на базе теории 2-го порядка. Поперечное перемещение в области малых волновых чисел $\kappa<\kappa *$ описывается теорией 3-го порядка с достаточной точностью.

3. Теории 4-го и 5-го порядка обеспечивают не только близкую к точной форму второй дисперсионной ветви [48], но и при малых значениях волнового числа хорошую аппроксимацию формы продольного перемещения, практически неотличимую от аппроксимаций высших порядков при $c_{g}^{\min }$ и $c_{g}=0$. Следовательно, теория 4-го порядка может считаться достаточно точной приближенной моделью для описания поля перемещений в области проявления эффекта «обратной волны». Теория 5-го порядка при волновых числах, не превышающих $\kappa *$, не предоставляет заметных преимуществ по сравнению с теорией 4-го порядка.

4. При увеличении волнового числа до значений $2 \kappa / \pi \approx 1$, т. е. при описании «прямой» волны в области, непосредственно прилежащей к диапазону волновых чисел, где проявляется эффект «обратной» волны, точность теорий 4-5-го порядков представляется также достаточной для решения практических задач. При дальнейшем увеличении волнового числа теория 5-го порядка не может описать распределение перемещений, соответствующих второй распространяющейся моде, с достаточной точностью: при $2 \kappa / \pi \approx 1,5$ требуется применение теорий 6-7-го порядков для достижения хорошей аппроксимации продольного перемещения $u_{1}^{2}$.

На основе перечисленных выводов представляется возможным заключить, что для описания явления «обратной волны» в упругом слое теориями пластин типа И.Н. Векуа А.А. Амосова, основанных на ортогональных разложениях неизвестных по базису, образованному полиномами Лежандра, минимально необходимыми порядками теории являются 4-5-й порядки, обеспечивающие точность построения как дисперсионной ветви, так и распределения перемещений по толщине слоя при распространении второй продольной моды прогрессивных волн.

Работа выполнена при финансовой поддержке РФФИ (проекты № 13-01-00446_a, № 14-01-00488_a, № 14-01-00890_a). 


\section{Библиографический список}

1. Kienzler R., Altenbach H., Otts I. Theories of Plates and Shells: Critical Review and New Applications. - Berlin: Springer-Verlag, 2004.

2. Eremeyev V., Pietraszkiewicz W. Refined theories of plates and shells // Zeitschrift für Angewandte Mathematik und Mechanik. - 2014. - Vol. 94(1-2). - P. 5-6.

3. Галиньш А. К. Расчет пластин и оболочек по уточненным теориям // Исследования по теории пластин и оболочек. - Вып. 6-7. - Казань: Изд-во Казан. ун-та, 1970. - С. 23-64.

4. Григолюк Э.И., Селезов И.Т. Итоги науки и техники. МДТТ. Т.5. Неклассические теории колебаний стержней, пластин и оболочек. - М.: ВИНИТИ, 1973. - 273 с.

5. Carrera E. A study of transverse normal stress effect on vibration of multilayered plates and shells // J. Sound \& Vibration. - 1999. - Vol. 225(5). - P. 803-829.

6. Carrera E. A Reissner's mixed variational theorem applied to vibration analysis of multilayered shell // Trans. ASME. J. Appl. Mech. - 1999. - Vol. 66. - P. 69-78.

7. Carrera E. Theories and Finite Elements for Multilayered, Anisotropic, Composite Plates and Shells // Arch. Comput. Methods Eng. - 2002. - Vol. 9(2). - P. 87-140.

8. Koiter W.T. A consistent first approximations in the general theory of thin elastic shells // Proc. of Symp. on the Theory of Thin Elastic Shells. August 1959, North Holland, Amsterdam. - P. 12-23.

9. Carrera E., Brischetto S. Analysis of thickness locking in classical, refined and mixed multilayered plate theories // Composite Structures. - 2008. - Vol. 82. - P. 549-562.

10. Brichetto S, Carrera S. Importance of Higher Order Modes and Refined Theories in in Free Vibration Analysis of Composite Plates // J. Appl. Math. - 2010. - Vol. 77(1), 011013 (14).

11. Batra R.C., Aimmanee S. Vibrations of thick isotropic plates with higher order shear and normal deformable plate theories // Comput. and Struct. - 2005. - Vol. 83. - P. 934-955.

12. Three-dimensional vibration analysis of circular and annular plates via the Chebyshev-Ritz method / D. Zhou, F.T.K. Au, Y.K. Cheung, S.H. Lo // Int. J. Sol. Struct. - 2003. - Vol. 40. - P. 3089-3105.

13. Kang Jae-Hoon, Leissa A. W. Three-Dimensional Vibration Analysis of Thick, Complete Conical Shells // Trans. of ASME. - 2004. - Vol. 71(6). - P. 502-507.

14. Matsunaga $H$. Stress analysis of functionally graded plates subjected to thermal and mechanical loadings // Compos. Struct. - 2009. - Vol. 87. - P. 344-357.

15. Matsunaga $\mathrm{H}$. Free vibration and stability of functionally graded plates according to a $2 \mathrm{D}$ higher-order deformation theory // Compos. Struct. - 2008. - Vol. 82. - P. 499-512.

16. Matsunaga $\mathrm{H}$. Thermal buckling of functionally graded plates according to a $2 \mathrm{D}$ higher-order deformation theory // Compos. Struct. - 2009. - Vol. 90. - P. 76-86.

17. Matsunaga $\mathrm{H}$. Free vibration and stability of functionally graded shallow shells according to a 2D higher-order deformation theory // Compos. Struct. - 2008. - Vol. 84. - P. 132-146.

18. Matsunaga $H$. Free vibration and stability of functionally graded circular cylindrical shells according to a 2D higher-order deformation theory // Compos.Struct. - 2009. - Vol. 88. - P. 519-531.

19. Абросимов Н.А., Баженов В.Г., Куликова Н.А. Идентификация вязкоупругих характеристик композитных материалов по результатам экспериментально- теоретического анализа динамического поведения полусферических оболочек // ПМТФ. - 2006. - Т. 47, № 3. - С. 126-133.

20. Абросимов Н.А., Куликова Н.А. Расчетно-экспериментальный метод идентификации вязкоупругих характеристик композитных материалов в динамически нагруженных оболочках вращения // Механика композит. материалов. - 2007. - Т.43, № 4. - С. 449-464.

21. Абросимов Н.А., Куликова Н.А. Определение параметров моделей нелинейного деформирования изотропных и композитных материалов по результатам расчетно- экспериментального анализа импульсного нагружения круглых пластин // ПМТФ. - 2011. - Т. 52, № 1. - С. 163-172.

22. Абросимов Н.А., Куликова Н.А. Идентификация параметров моделей вязкоупругого деформирования композитных материалов по результатам расчетно-экспериментального анализа импульсного нагружения оболочек вращения // Изв. РАН. МТТ. - 2011. - № 3. - С. 42-57. 
23. Ардзашвили Р.В., Вильде М.В., Коссович Л.Ю. Антисимметричные кромочные волны высшего порядка в пластинах // Изв. Саратов. ун-та. Нов. сер. Математика, механика, информатика. - 2013. - Вып. 1. Ч. 1. - Т. 13. - С. 51-56.

24. Кильчевский Н.А. Основы аналитической механики оболочек. - Киев: Изд-во АН УССР, 1963. $-634 \mathrm{c}$.

25. Ворович И.И. Некоторые математические задачи теории пластин и оболочек // Tp. II Bceсоюзн. съезда по теор. и прикл. мех., 1964. - М.: Наука, 1966. - С. 116-136.

26. Гольденвейзер А.Л. Построение приближенной теории оболочек при помощи асимптотического интегрирования уравнений теории упругости // ПММ. - 1963. - Т. 27, № 4. - С. 593-608.

27. Коссович Л.Ю. Асимптотические методы в динамике оболочек при ударных воздействиях // Изв. Сарат. ун-та. Сер. Математика, механика, информатика. - 2008. - Т. 8. - Вып. 2. - С. 12-33.

28. Zhilin P.A. Mechanics of Deformable Cosserat Surfaces // Int. J. Sol. Struct. - 1976. - Vol. 12. P. 635-648.

29. Жилин П.А. Прикладная механика: основы теории оболочек: учеб. пособие. - СПб.: Издво С.-Петерб. политехн. ун-та, 2006. - 167 с.

30. Yokoo Y., Matsunaga H. A general nonlinear theory of elastic shells // Int. J. Sol. Struct. 1974. - Vol. 10. - P. 261-274.

31. Векуа И.Н. Некоторые общие методы построения различных вариантов теории оболочек. М: Наука, 1982. -282 с.

32. Гуляев В.И., Баженов В.А., Лизунов П.П. Неклассическая теория оболочек и ее приложение к решению инженерных задач. - Львов: Вища Школа, 1978. - 192 с.

33. Хома И.Ю. Общая теория анизотропных оболочек. - Киев: Наук. думка, 1986. - 170 с.

34. Абросимов Н.А., Баженов В.Г. Исследование динамического деформирования упругопластических сферических оболочек при тепловом ударе // Изв. АН СССР. МТТ. - 1978. - № 1. C. $139-143$.

35. Абросимов Н.А., Баженов В.Г. Нелинейные задачи динамики композитных конструкций. Н. Новгород: Изд-во Нижегород. гос. ун-та, 2002. - 400 с.

36. Никабадзе М.У. Развитие метода ортогональных полиномов в механике микрополярных и классических упругих тонких тел. - М.: Изд-во МГУ, 2014. - 515 с.

37. Амосов А.А. Приближенная трехмерная теория толстостенных пластин и оболочек // Строительная механика и расчет сооружений. - 1987. - № 5. - С. 37-42.

38. Амосов А.А., Жаворонок С.И. К проблеме редукции плоской задачи теории упругости к последовательности одномерных краевых задач // Мех. композ. матер. и констр. - 1997. - Т. 3 , № 1. - C. 69-80.

39. Амосов А.А., Князев А.А., Жаворонок С.И. О решении некоторых краевых задач о плоском напряженном состоянии криволинейной трапеции // Механика композ. матер. и констр. 1999. - Т. 5, № 1. - С. 60-72.

40. Amosov A.A., Zhavoronok S.I. An approximate high-order theory of thick anisotropic shells // Int. J. Comput. Civil Struct. Eng. - 2003. - Vol. 1. - P. 28-38.

41. Амосов А.А., Жаворонок С.И., Леонтьев К.А. О решении некоторых задач о напряженнодеформированном состоянии анизотропных толстостенных оболочек вращения в трехмерной постановке // Мех. композ. матер. и констр. - 2004. - Т. 10, № 3. - С. 301-310.

42. Жаворонок С. И. Модели высшего порядка анизотропных оболочек // Механика композ. матер. и констр. - 2008. - Т. 14, № 4. - С. 561-571.

43. Жаворонок С.И., Леонтьев А.Н., Леонтьев К.А. Анализ сходимости решения при расчете толстостенных оболочек вращения произвольной формы // Int. J. Comput. Civil Struct. Eng. 2010. - Vol. 6(1-2). - P. 105-111.

44. Кильчевский Н.А., Кильчинская Г.А., Ткаченко Н.Е. Аналитическая механика континуальных систем. - Киев: Наукова Думка, 1979. - 189 с. 
45. Жаворонок С.И. Вариационные уравнения трехмерной теории анизотропных оболочек // Вестн. Нижегород. ун-та им. Н.И. Лобачевского. - 2011. - № 4(5). - С. 2153-2155.

46. Жаворонок С.И. Обобщенные уравнения Лагранжа второго рода трехмерной теории анизотропных оболочек // Мех. композ. матер. и констр. - 2011. - Т. 17, № 1. - С. 116-132.

47. Zhavoronok S.I. A Vekua-type linear theory of thick elastic shells // Zeitschrift fur Angewandte Mathematik und Mechanik. - 2014. - Vol. 94 (1-2). - P. 164-184.

48. Zhavoronok S.I. Variational formulations of Vekua-type shell theories and some their applications // Shell Structures: Theory and Applications. Vol. 3. CRC Press. - Balkema: Taylor \& Francis Gr., 2014. - P. 341-344.

49. Жаворонок С.И. Формулировка начально-краевой задачи приближенной трехмерной теории оболочек $\mathrm{N}$-го порядка в обобщенных перемещениях и ее приложение к задачам стационарной динамики // Мех. композ. матер. и констр. -2012. - Т. 18, № 3. - С. 333-344.

50. Пикуль В.В. Физически корректные модели материала упругих оболочек // Изв. РАН. MTT. - 1995. - № 2. - C. 103-108.

51. Schwab C., Wright S. Boundary layers in hierarchical beam and plate models // J. Elast. 1995. - Vol. 38. - C. 1-40.

52. Kulikov G.M, Plotnikova S.V. Equivalent single-layer and layerwise shell theories and rigidbody motions. Part I: Foundations // Mech. Adv. Mater. Struct. - 2005. - Vol. 12(4). - P. 275-283.

53. Жаворонок С.И. Исследование гармонических волн в упругом слое на основе трехмерной теории оболочек N-го порядка // Механика композиционных материалов и конструкций. - 2010. T. 16, № 4/2. - С. 693-701.

54. Жаворонок С.И. Трехмерные модели динамического деформирования толстостенных оболочек // Морские интел. технол. -2011. - № 3 (спец.). - С. 6-10.

55. Жаворонок С.И. Исследование распространяющихся мод гармонических волн в упругом слое на базе трехмерной теории оболочек N-го порядка // Мех. композ. матер. и констр. - 2011. Т. 17, № 2. - С. 278-287.

56. Жаворонок С.И. Исследование кинематики нормальных волн в упругом слое на основе трехмерной теории оболочек $\mathrm{N}$-го порядка для различных значений волновых чисел // Механика композ. матер. и констр. - 2012. - Т. 18, № 1. - С. 45-56.

57. Егорова О.В., Жаворонок С.И., Курбатов А.С. О приложении различных вариантов теории оболочек $\mathrm{N}$-го порядка к некоторым задачам о прогрессивных волнах // Изв. Тул. гос. ун-та. Сер. Технические науки. - 2014. - № 11. - Ч. 1. - С. 255-266.

58. Гринченко В.Т., Мелешко В.В. Гармонические колебания и волны в упругих телах. Киев: Наукова думка, 1981. - 284 с.

59. Meitzler A. H. Backward-wave transmission of stress pulses in elastic cylinders and plates // J. Acoust. Soc. Amer. - 1965. - Vol. 38(5). - P. 835-842.

60. Артоболевский И.И., Бобровницкий Ю.И., Генкин М.Д. Введение в акустическую динамику машин. - М: Наука, 1979. - 306 с.

\section{References}

1. Kienzler R., Altenbach H., Otts I. Theories of Plates and Shells: Critical Review and New Applications. Berlin: Springer-Verlag, 2004.

2. Eremeyev V., Pietraszkiewicz W. Refined theories of plates and shells. Zeitschrift für Angewandte Mathematik und Mechanik, 2014, vol. 94(1-2), pp. 5-6.

3. Galinsh A.K. Raschet plastin i obolochek po utochnennym theoriyam (Computation of plates and shells using advanced theories). Issledovaniia po teorii plastin i obolochek. Kazanskii universitet, 1970, iss. 6-7, pp. 23-64.

4. Grigoliuk E.I., Selezov I.T. Itogi nauki i tekhniki. Mekhanika deformiruemogo tverdogo tela. Vol. 5. Neklassicheskie teorii kolebanii sterzhnei, plastin i obolochek [Resumes of Science and Technics. 
Mechanics of Solids. Vol. 5. Non-classical theories of beams, plates and shells vibrations]. Moscow: VINITI, 1973. $273 \mathrm{p}$.

5. Carrera E. A study of transverse normal stress effect on vibration of multilayered plates and shells. J. Sound \& Vibration, 1999, vol. 225(5), pp. 803-829.

6. Carrera E. A Reissner's mixed variational theorem applied to vibration analysis of multilayered shell. Trans. ASME. J. Appl. Mech., 1999, vol. 66, pp. 69-78.

7. Carrera E. Theories and Finite Elements for Multilayered, Anisotropic, Composite Plates and Shells. Arch. Comput. Methods Eng., 2002, vol. 9(2), pp. 87-140.

8. Koiter W.T. A consistent first approximations in the general theory of thin elastic shells. Proc. of Symp. on the Theory of Thin Elastic Shells. August 1959. North Holland, Amsterdam, pp. 12-23.

9. Carrera E., Brischetto S. Analysis of thickness locking in classical, refined and mixed multilayered plate theories. Composite Structures, 2008, vol. 82, pp. 549-562.

10. Brichetto S., Carrera S. Importance of Higher Order Modes and Refined Theories in in Free Vibration Analysis of Composite Plates. J. Appl. Math., 2010, vol. 77(1), 011013 (14 pp).

11. Batra R.C., Aimmanee S. Vibrations of thick isotropic plates with higher order shear and normal deformable plate theories. Comput. and Struct., 2005, vol. 83, pp. 934-955.

12. Zhou D., Au F.T.K., Cheung Y.K., Lo S.H. Three-dimensional vibration analysis of circular and annular plates via the Chebyshev-Ritz method. Int. J. Sol. Struct., 2003, vol. 40, pp. 3089-3105.

13. Kang Jae-Hoon, Leissa A.W. Three-Dimensional Vibration Analysis of Thick, Complete Conical Shells. Trans. of ASME, 2004, vol. 71(6), pp. 502-507.

14. Matsunaga H. Stress analysis of functionally graded plates subjected to thermal and mechanical loadings. Compos. Struct., 2009, vol. 87, pp. 344-357.

15. Matsunaga H. Free vibration and stability of functionally graded plates according to a $2 \mathrm{D}$ higher-order deformation theory. Compos. Struct., 2008, vol. 82, pp. 499-512.

16. Matsunaga $\mathrm{H}$. Thermal buckling of functionally graded plates according to a $2 \mathrm{D}$ higher-order deformation theory. Compos. Struct., 2009, vol. 90, pp. 76-86.

17. Matsunaga H. Free vibration and stability of functionally graded shallow shells according to a 2D higher-order deformation theory. Compos. Struct., 2008, vol. 84, pp. 132-146.

18. Matsunaga H. Free vibration and stability of functionally graded circular cylindrical shells according to a 2D higher-order deformation theory. Compos. Struct., 2009, vol. 88, pp. 519-531.

19. Abrosimov N.A., Bazhenov V.G., Kulikova N.A. Identifikatsiia viazkouprugikh kharakteristik kompozitnykh materialov po rezul'tatam eksperimental'no-teoreticheskogo analiza dinamicheskogo povedeniia polusfericheskikh obolochek [Identification of viscoelastic characteristics of composite materials on the basis of results of an experimental and theoretical analysis of the dynamic behavior of hemispherical shells]. J. Appl. Mech. Techn. Phys., 2006, vol. 47(3), pp. 412-418.

20. Abrosimov N.A., Kulikova N.A. Raschetno-eksperimental'nyi metod identifikatsii viazkouprugikh kharakteristik kompozitnykh materialov $\mathrm{v}$ dinamicheski nagruzhennykh obolochkakh vrashcheniia [A computational-experimental method for identifying the viscoelastic properties of composites in dynamically loaded shells of revolution]. Mechanics of Compos. Mater., 2007, vol. 43(4), pp. 449-464.

21. Abrosimov N.A., Kulikova N.A. Opredelenie parametrov modelei nelineinogo deformirovaniia izotropnykh i kompozitnykh materialov po rezul'tatam raschetno-eksperimental'nogo analiza impul'snogo nagruzheniia kruglykh plastin [Determining the model parameters of nonlinear deformation of isotropic and composite materials from the results of an experimental-computational analysis of impulsively loaded circular plates]. J. Appl. Mech. Techn. Phys., 2011, vol. 52(1), pp. 132-140

22. Abrosimov N.A., Kulikova N.A. Parameter identification in viscoelastic strain models for composite materials by analyzing impulsive loading of shells of revolution. Mechanics of Solids: A Journal of Russian Academy of Sciences, 2011, vol. 46(3), pp. 368-379.

23. Ardzashvili R.V., Wilde M.V., Kossovich L.Yu. Antisimmetrichnye kromochnye volny vyschego poriadka $\mathrm{v}$ plastinakh [High-order boundary waves in plates]. Izvestiia Saratovskogo universiteta. Mathematika, Mechanika, Informatika, 2013, vol. 13, iss. 1, part 1, pp. 51-56. 
24. Kilchevskiy N.A. Osnovy analiticheskoi mekhaniki obolochek [Basics of analytical shell mechanics]. Kiev: Ukrainian Acad. Sci., 1963. 634 p.

25. Vorovich I.I. Nekotorye matematicheskie zadachi teorii plastin i obolochek [Some mathematical problems of plate and shell theory]. Proc. of II All-Union Conf. on Theoretical and Applied Mechanics, 1964. Review. Moscow: Nauka, 1966, pp. 116-136.

26. Goldenweiser A.L. Postroenie priblizhennoi teorii obolochek pri pomoshchi asimptoticheskogo integrirovaniia uravnenii teorii uprugosti [Derivation of an approximate theory of shells by means of asymptotic integration of the equations of the theory of elasticity]. Appl. Math. Mech., 1963, vol. 27(4), pp. 903-924.

27. Kosovich L.Yu. Asimptoticheskie metody v dinamike obolochek pri udarnykh vozdeistviakh [Asymptotic Methods in Dynamics of Shells under Shock Loading]. Izvestiia Saratovskogo universiteta. Mathematika, Mechanika, Informatika, 2008, vol. 8, iss. 2, pp. 12-33.

28. Zhilin P.A. Mechanics of Deformable Cosserat Surfaces. Int. J. Sol. Struct., 1976, vol. 12, pp. 635-648.

29. Zhilin P.A. Prikladnaia mekhanika: osnovy teorii obolochek [Applied Mechanics: Basics of Shells' Theory]. St. Petersburgskii polytechnicheskii universitet, 2006. $167 \mathrm{p}$.

30. Yokoo Y., Matsunaga H. A general nonlinear theory of elastic shells. Int. J. Sol. Struct., 1974, vol. 10, pp. 261-274.

31. Vekua I.N. Nekotorye obshchie metody postroenia razlichnykh variantov teorii obolochek [Some general methods of construction of various variants of theory of shells]. Moscow: Nauka, 1982. 282 p.

32. Guliaev V.I., Bazhenov V.A., Lizunov P.P. Neklassicheskaia teoria obolochek i ee prilozhenie k resheniiu inzhenernykh zadakh [Non-classical shell theory and its application to solving of enrineering problems]. Lwov: Vischa Shkola, 1978. 192 p.

33. Khoma I.Yu. Obschaia teoriia anizotropnykh obolochek [General Theory of Anizotropic Shells]. Kiev: Naukova Dumka, 1986. 170 p.

34. Abrosimov N.A., Bazhenov V.G. Issledovanie dinamicheskogo deformirovaniia uprugoplasticheskikh sfericheskikh obolochek pri teplovom udare [Investigation of the dynamic strain state of elastic-plastic spherical shells under heat impact]. Mechanics of Solids: A Journal of Russian Academy of Sciences, 1978, no. 1, pp. 139-143.

35. Abrosimov N.A., Bazhenov V.G. Nelineinye zadachi dinamiki kompositnykh konstrukcii [Nonlinear Problems of Dynamics of Composite Structures]. Nizhegorodskii universitet, 2002. 400 p.

36. Nkabadze M.U. Razvitie metoda ortogonalnykh polinomov $\mathrm{v}$ mekhanike mikropolarnykh i klassicheskikh uprugikh tonkikh tel [Development of the Orthogonal Polynomials Method in the Mechanics of Micropolar and Traditional Elastic Bodies]. Moscovskii gosudarstvennyi universitet, 2014. $515 \mathrm{p}$.

37. Amosov A.A. Priblizhennaia trekhmernaia teoriia tolstostennykh plastin i obolochek [An approximated three-dimensional theory of thick-walled shells and plates]. Stroitelnaia mekhanika $i$ raschet sooruzhenii, 1987, no. 5, pp. 37-42.

38. Amosov A.A., Zhavoronok S.I. K probleme reduktsii ploskoi zadachi teorii uprugosti $\mathrm{k}$ posledovatel'nosti odnomernykh kraevykh zadach [Reduction of the plane problem of elasticity theory to a sequence of one-dimensional boundary-value problems]. Composite Mechanics and Design, 1997, vol. 3(1), pp. 69-80.

39. Amosov A.A., Kniazev A.A., Zhavoronok S.I. On solution of 2D-problem of stressed curvilinear trapezoid. Composite Mechanics and Design, 1999, vol. 5(1), pp. 60-72.

40. Amosov A.A., Zhavoronok S.I. An approximate high-order theory of thick anisotropic shells. Int. J. Comput. Civil Struct. Eng., 2003, vol. 1, pp. 28-38.

41. Amosov A.A., Zhavoronok S.I., Leontiev K.A. O reshenii nekotorykh zadach o napriazhennodeformirovannom sostoianii anizotropnykh tolstostennykh obolochek vrashcheniia $\mathrm{v}$ trekhmernoi postanovke [On the solution of some problems of stress-strain state of thick anisotropic shells of revolution on the basis of the three-dimensional problem's statement]. Compos. Mech. and Design, 2004, vol. 10(3), pp. 301-310. 
42. Zhavoronok S.I. Modeli vysshego poriadka anizotropnykh obolochek [High-order anisotropic shells models]. Composite Mechanics and Design, 2008, vol. 14(4), pp. 561-571.

43. Zhavoronok S.I., Leontiev A.N., Leontiev K.A. Analysis of thick-walled rotation shells based on Legendre polynomials. Int. J. Comput. Civil Struct. Eng., 2010, vol. 6(1-2), pp. 105-111.

44. Kilchevskiy N.A., Kilchinskaia G.A., Tkachenko N.E. Analiticheskaia mekhanika kontinualnykh system [Analytical mechanics of continual systems]. Kiev: Naukova Dumka, 1979. 189 p.

45. Zhavoronok S.I. Variacionnye uravnenia trekhmernoi teorii anizotropnykh obolochek [Variational equations of the three-dimensional theory of anisotropic shells]. Vestnik Nizhegorodskogo universiteta imeni N.I. Lobachevskogo, 2011, vol. 4(5), pp. 2153-2155.

46. Zhavoronok S.I. Obobshchennye uravneniia Lagranzha vtorogo roda trekhmernoi teorii anizotropnykh obolochek [Generalized Lagrange equations of the second kind of three-dimensional anisotropic shell theory]. Composite Mechanics and Design, 2011, vol. 17(1), pp. 116-132.

47. Zhavoronok S.I. A Vekua-type linear theory of thick elastic shells. Zeitschrift fur Angewandte Mathematik und Mechanik, 2014, vol. 94 (1-2), pp. 164-184.

48. Zhavoronok S.I. Variational formulations of Vekua-type shell theories and some their applications. Shell Structures: Theory and Applications, vol. 3. CRC Press. Balkema: Taylor \& Francis Gr., 2014. 341-344 p.

49. Zhavoronok S.I. Formulirovka nachal'no-kraevoi zadachi priblizhennoi trekhmernoi teorii obolochek Ngo poriadka $\mathrm{v}$ obobshchennykh peremeshcheniiakh i ee prilozhenie $\mathrm{k}$ zadacham statsionarnoi dinamiki [A formulation of the three-dimensional approximated shells theory of $\mathrm{n}$-th order using generalized displacements and its application to steady dynamics]. Composite Mechanics and Design, 2012, vol. 18(3), pp. 333-344.

50. Pikul V.V. Fizicheski korrektnye modeli materiala uprugikh obolochek [Physically correct material models for elastic shells]. Mechanics of Solids: A Journal of Russian Academy of Sciences, 1995, no. 2, pp. 103-108.

51. Schwab C., Wright S. Boundary layers in hierarchical beam and plate models. J. Elast., 1995, vol. 38, pp. 1-40.

52. Kulikov G.M, Plotnikova S.V. Equivalent single-layer and layerwise shell theories and rigidbody motions. Part I: Foundations. Mech. Adv. Mater. Struct., 2005, vol. 12(4), pp. 275-283.

53. Zhavoronok S.I. Issledovanie garmonicheskikh voln $\mathrm{v}$ uprugom sloe na osnove trekhmernoi teorii obolochek N-go poriadka [Investigation of harmonic waves in elastic layer using N-th order threedimensional shells theory]. Composite Mechanics and Design, 2010, vol. 16(4/2), pp. 693-701.

54. Zhavoronok S.I. Trekhmernye modeli dinamicheskogo deformirovania tolstostennykh obolochek [Three-dimensional models of dynamical deformed state of thick-walled shells]. Morskie intellektualnye tekhnologii, 2011, vol. 3, pp. 6-10.

55. Zhavoronok S.I. Issledovanie rasprostraniaiushchikhsia mod garmonicheskikh voln v uprugom sloe na baze trekhmernoi teorii obolochek N-go poriadka [Investigation of propagating modes of harmonic waves in elastic layer using $\mathrm{N}$-th order three-dimensional shells theory]. Comp.Mech.and Design, 2011, vol. 17(2), pp. 278-287.

56. Zhavoronok S.I. Kinematics of normal modes in elastic layer for some wavenumbers investigation based on N-th order three-dimensional shells' theory. Composite Mechanics and Design, 2012, vol. $18(1)$, pp. $45-56$.

57. Egorova O.V., Zhavoronok S.I., Kurbatov A.S. O prilozhenii razlitchnykh variantov teorii obolochek N-go poriadka k nekotorym zadacham o progrssivnykh volnakh [On the application of different variants of N'th order shell theory to some problems of normal waves propagation]. Part 1. Izvestiia Tulskogo gosudarstvennogo universiteta. Technical Sci., 2014, no. 11, pp. 255-266.

58. Grinchenko V.T., Meleshko V.V. Garmonicheskie kolebaniia i volny v uprugikh telakh [Harmonic waves in elastic bodies]. Kiev: Naukova dumka, 1981. 284 pp.

59. Meitzler A.H. Backward-wave transmission of stress pulses in elastic cylinders and plates. J. Acoust. Soc. Amer., 1965, vol. 38(5), pp. 835-842.

60. Artobolevskiy I.I., Bobrovnitskiy Yu.I., Genkin M.D. Vvedenie v akusticheskuiu dinamiku mashin [Introduction into the acoustical machine dynamics]. Moscow: Nauka, 1979. 306 p. 